\title{
UPSILON SPECTROSCOPY: Transitions in the Bottomonium System
}

\author{
D. Besson
}

Department of Physics, University of Kansas, Lawrence, Kansas 66045

\section{T. Skwarnicki}

Department of Physics, Southern Methodist University, Dallas, Texas 75275

KEY WORDS: potential models, radiative transitions, hadronic transitions, multiple models

\section{CONTENTS}

1. INTRODUCTION . . ............................... 334

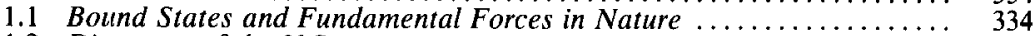

1.2 Discovery of the $Y$ System ..................................... 335

1.3 The Quarkonium Model ................................ 335

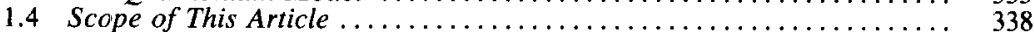

2. STORAGE RINGS AND DETECTORS $\ldots \ldots \ldots \ldots \ldots \ldots \ldots \ldots \ldots \ldots . \ldots \ldots$

3. COUPLING TO LEPTON PAIRS ........................ 339

3.1 Measurement of $Y$ Masses and Their Leptonic Widths ............. 339

3.2 Measurement of Leptonic Branching Ratios .................... 341

3.3 Derivation of the Total Widths of the Y Resonances $\ldots \ldots \ldots \ldots \ldots \ldots, 342$

3.4 Comparison with Theoretical Predictions ................... 342

4. MEASUREMENTS OF RADIATIVE TRANSITIONS $\ldots \ldots \ldots \ldots \ldots \ldots .343$

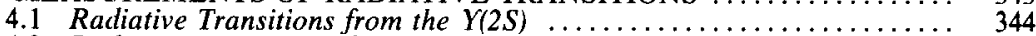

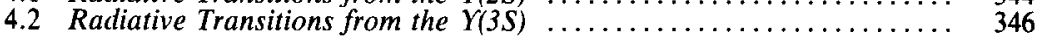

5. COMPARISON WITH POTENTIAL MODELS $\ldots \ldots \ldots \ldots \ldots \ldots \ldots \ldots, 348$

5.1 Excitation Energies ................................... 348

5.2 Electric Dipole Matrix Elements ....................... 353

5.3 Hadronic Widths of the P States ................................... 356

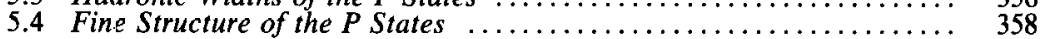

6. HADRONIC TRANSITIONS BETWEEN BOTTOMONIA $\ldots \ldots \ldots \ldots \ldots, 360$

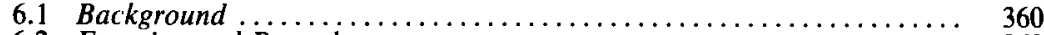

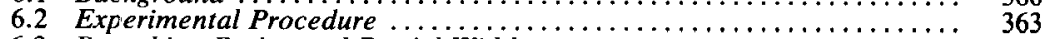

6.3 Branching Ratios and Partial Widths ............................... 363

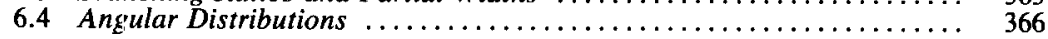


6.5 Dipion Mass Spectra ............................... 366

6.6 Unanswered Questions .......................................... 370

7. DIRECT DECAYS OF BOTTOMONIA INTO HADRONS, QUARK vs

GLUON FRAGMENTATION $\ldots \ldots \ldots \ldots \ldots \ldots \ldots \ldots \ldots \ldots \ldots, \quad 370$

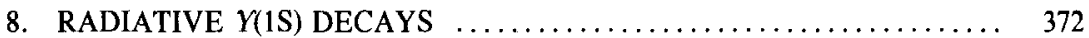

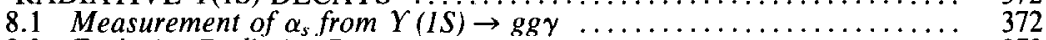

8.2 Exclusive Radiative Decays ................................ 373

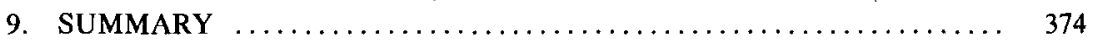

\section{INTRODUCTION}

\subsection{Bound States and Fundamental Forces in Nature}

At astronomical distances objects are held together by gravitational forces. At distances of the order of $10^{-10} \mathrm{~m}$, the electromagnetic force dominates, binding electrons to the nucleus. Peripheral electromagnetic interactions bind atoms into molecules or even larger structures. The simplest atom consists of just one electron bound to a nucleus in a nonrelativistic two-body system.

At distances of the order of $10^{-15} \mathrm{~m}$, similar structure is created by the strong interactions. Strong forces bind quarks into nucleons and peripheral strong interactions bind nucleons into nuclei. Unlike the simplest atom, a nucleon is a complicated three-body system. Twobody systems of quark-antiquark pairs (e.g. the $\pi$ meson) were discovered, but they were still complicated by the relativistic velocities of quarks and by the existence of three different quarks $(u, d, s)$ with approximately equal effective masses. The complexity of the lightest systems bound by strong interactions obscured the nature of strong forces and the quark structure of matter for a long time.

The discovery of the bound system of heavy charm-anticharm quarks $(\psi$ states-c $\overline{\mathrm{C}})$ in 1974 (1) was a turning point in the development of the theory of quarks and their interactions. Even heavier and more nonrelativistic $Y$ states were discovered in $1977(2)$. These $Y$ resonances were identified as bound states of $b \bar{b}$ quark pairs $(b$ is the bottom quark).

The $Y$ particles are about ten times heavier than the nucleon and about three times heavier than the $\psi$ particles. While light quark systems are completely relativistic, $(v / c)^{2} \approx 1$, the $Y$ system is nearly nonrelativistic, $(v / c)^{2} \approx 0.08$. Since nonrelativistic systems are easier to describe theoretically, the $Y$ system plays an important role in studies of strong interactions.

Compared to the $\psi$ family $(v / c)_{\psi}^{2} \approx 0.24$, the $Y$ system offers a larger number of long-lived states bound in a less relativistic system. The standard model predicts the existence of yet another, more nonrela- 
tivistic quarkonia state, "toponium," consisting of the sixth quark called "top" (t) and its antiparticle. So far, experimental searches sensitive to quark masses up to about 20 times the $b$ quark mass have not revealed the top (3). Estimates based on higher order corrections to observed $Z^{0}$ decay rates indicate a bare top mass of order 110-190 $\mathrm{GeV}$. If discovered, the $\mathrm{t}_{\mathrm{t}}$ system will be completely nonrelativistic. However, experimental studies of strong interaction phenomena in the $\bar{t} \bar{t}$ states will be very difficult, if not impossible, because the top quark will decay by weak interactions at a rate comparable to that of strong interactions (4). This leaves the $Y$ system the simplest strongly bound system among all quarkonia.

\subsection{Discovery of the $Y$ System}

The $Y$ system was discovered in the collision of a beam of high energy protons with a stationary nuclear target (2). The invariant mass distribution of $\mu^{+} \mu^{-}$pairs produced in these collisions revealed peaks interpreted as three hadronic resonances, $Y, Y^{\prime}, Y^{\prime \prime}$, decaying into two muons. The existence of these states was soon confirmed in the inverse of this process, $\mathrm{e}^{+} \mathrm{e}^{-} \rightarrow Y^{(n)} \rightarrow$ light quark states (5). In this process, the entire collision energy of the initial electron and positron turns into the rest mass of the $Y$ state. The beam energy must be matched to the resonance mass; thus only one $Y$ resonance may be directly produced at a time. On the other hand, the lack of any beam or production fragments produces a particularly clean environment for studies of the properties of the $Y$ states. So far, our knowledge of the properties of these states comes primarily from $\mathrm{e}^{+} \mathrm{e}^{-}$storage rings. Scans of the cross section of $\mathrm{e}^{+} \mathrm{e}^{-}$annihilation into light hadrons as a function of the $\mathrm{e}^{+} \mathrm{e}^{-}$center-of-mass energy revealed up to three more $Y$ states in addition to those observed in the hadronic production experiments, as shown in Figure 1 (6).

\subsection{The Quarkonium Model}

The formation of the $Y$ resonances in $\mathrm{e}^{+} \mathrm{c}^{-}$annihilation is mediated by a virtual photon $\mathrm{e}^{+} \mathrm{e}^{-} \rightarrow \gamma^{*} \rightarrow \mathrm{b} \overline{\mathrm{b}}$; thus these states must have the quantum numbers of a photon: $J^{P C}=1^{--}$.

The first three $Y$ resonances are observed with widths equal to the experimental error (i.e. beam energy spread at $\mathrm{e}^{+} \mathrm{e}^{--}$colliders). Their natural widths, related to their lifetimes, are derived indirectly (see Section 3) and are of the order of $25-50 \mathrm{keV}$. By contrast, the fourth $Y$ and subsequent resonance peaks are broader than the experimental resolution. The natural width of the fourth $Y$, for example, is 24,000 $\mathrm{keV}$. 


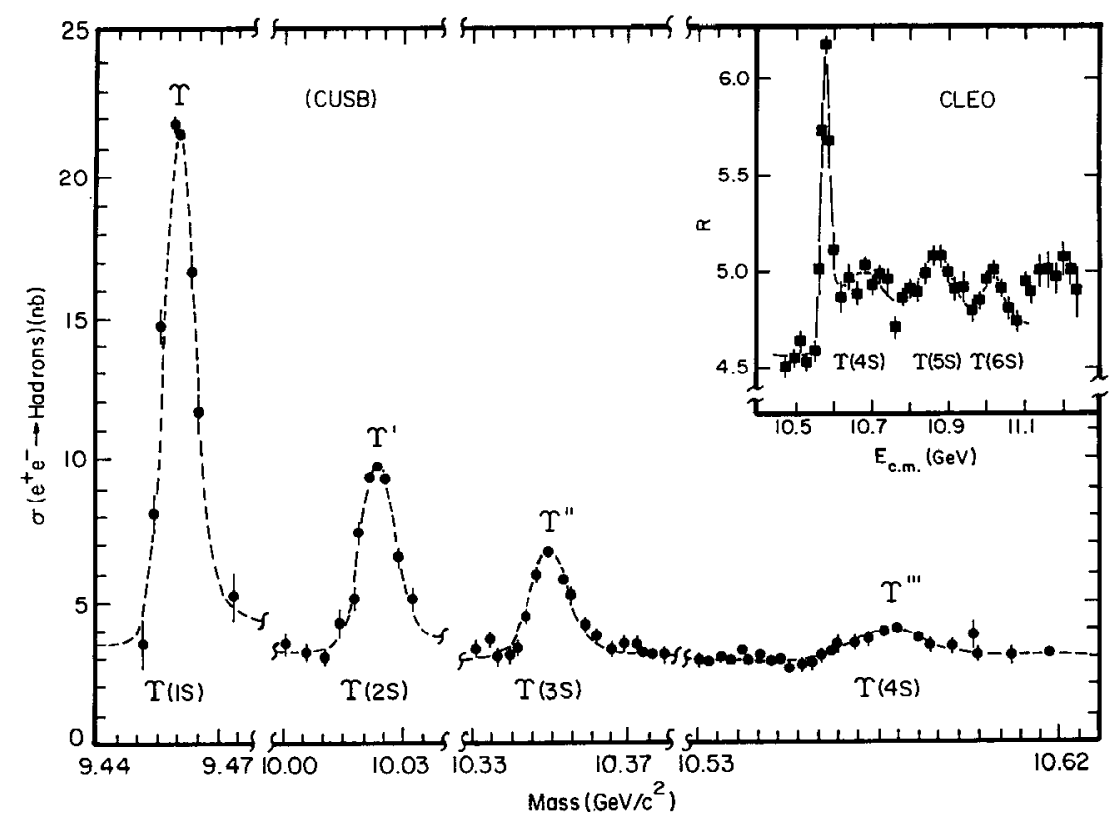

Figure I Cross section for inclusive production of hadrons as a function of center-ofmass energy.

This dramatic difference in the natural widths of the $Y$ 's is explained by the quarkonium model, which says that the $Y$ 's are bound states of the $b \bar{b}$ pairs. If kinematically allowed, these states decay by a typical strong interaction process into a pair of heavy-light quark mesons: $(b \bar{b})$ $\rightarrow(b \bar{q})(q \bar{b})(q=u$ or $d)$, i.e. $Y \rightarrow B \bar{B}$, which results in a large natural width. The first three $Y$ s have masses below the kinematic threshold for this decay. They can still decay by strong interactions if the $b \bar{b}$ pair annihilates into three gluons: $(b \bar{b}) \rightarrow 3 g \rightarrow$ hadrons. The quark annihilation process is slower by many orders of magnitude than the decays into $\mathrm{B} \overline{\mathrm{B}}$ pairs.

Because the $b \bar{b}$ states below $B \bar{B}$ threshold are long-lived and nonrelativistic, they create a positronium-like system bound by strong interactions. A variety of bound states corresponding to different radial (quantum number $n=1,2, \ldots$ ) and orbital momentum (quantum number $L=\mathrm{S}, \mathrm{P}, \mathrm{D}, \ldots$ ) excitations, and to different configurations of the quark spins (total spin $S=0,1$ ) are expected.

Since the system is nonrelativistic, the mass of each state depends primarily on $n$ and $L$. Relativistic effects generate fine splittings (as a result of spin-orbit and tensor interactions) and hyperfine splittings 
(arising from spin-spin interactions). The spin-parity of a given mass level is determined by its intrinsic quantum numbers: $|L-S| \leq J \leq$ $|L+S|, P=(-1)^{L+1}, C=(-1)^{L+S}$. We use here the spectroscopic symbol $n^{2 S+1} L_{J^{P C}}$ to denote different $\mathrm{b} \overline{\mathrm{b}}$ levels (on different occasions we drop some of these symbols for brevity). The masses of the $b \bar{b}$ states can be reproduced in a familiar potential model approach, as we discuss below. The mass spectrum predicted by one of the potential models is shown in Figure 2.

Among the states below $\mathrm{B} \overline{\mathrm{B}}$ threshold, only the $n^{3} \mathrm{~S}_{1^{--}}$and $n^{3} \mathrm{D}_{1^{-}-}$states have quantum numbers that match those of a photon. The strength of the coupling to a virtual photon is proportional to the value of the radial wave function at the origin, which is nonzero only for the $S_{1}$ states. Therefore, the $Y$ states discovered in the dimuon spectrum in the hadronic production and in the $\mathrm{e}^{+} \mathrm{e}^{-}$annihilation cross section must correspond to subsequent radial excitations of the triplet S states: $Y(1 S), Y(2 S), Y(3 S), Y(4 S), \ldots$.

Because strong decays of the $b \bar{b}$ states below $B \bar{B}$ threshold are suppressed, electromagnetic interactions become competitive and radiative transitions among various excitations of the $\bar{b} \bar{b}$ system are ob-

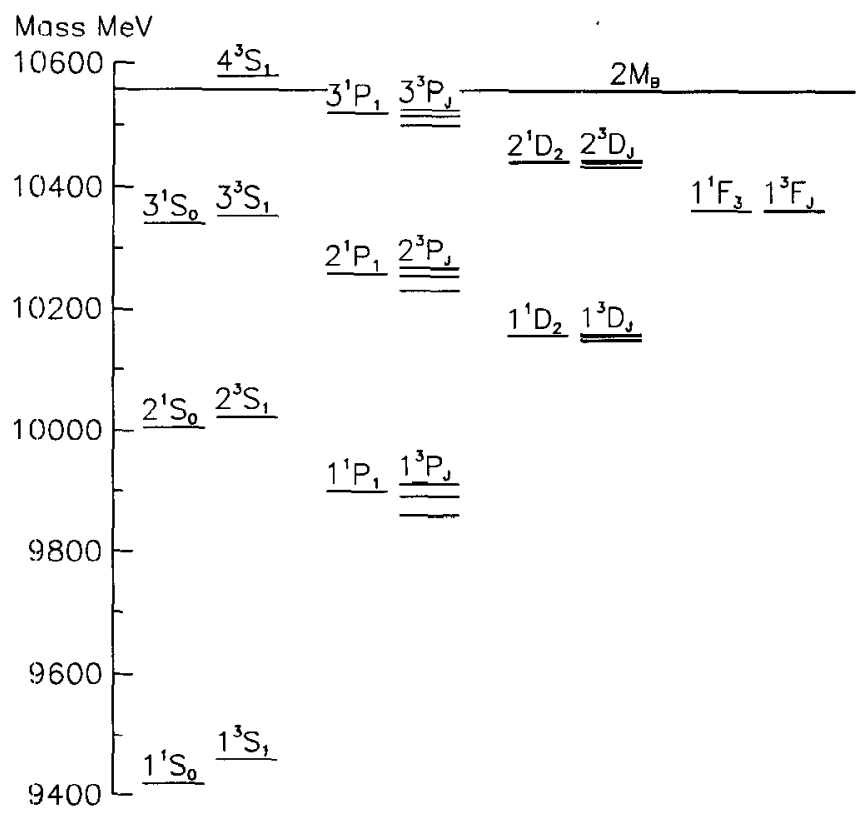

Figure 2 Mass spectrum of $\mathrm{b} \overline{\mathrm{b}}$ bound states below the $\mathrm{B} \overline{\mathrm{B}}$ threshold predicted by one of the potential models (75). 


\section{8}

\section{BESSON \& SKWARNICKI}

served. The excitation energy can also be released by emission of soft gluons, which turn into soft hadrons $\left(\pi^{0}, \eta, \omega, \pi \pi, \pi \pi \pi\right)$, without any change of the quark content of the bound state. Transitions from the $n^{3} \mathrm{~S}_{1-}$ - states to the lower states thereby allow the production and study at $\mathrm{e}^{+} \mathrm{e}^{-}$machines of $\mathrm{b} \overline{\mathrm{b}}$ states with different quantum numbers.

\subsection{Scope of This Article}

The last experimental review of the $r$ system in this journal appeared ten years ago (7). Since then, experimental knowledge about these states has improved significantly. We review this progress, with emphasis on recent results. Topics that are amply covered in other reviews are treated only briefly here. We recall the theoretical aspects of the hcavy quarkonium system mainly to summarize our present understanding of the data.

After a brief discussion of the experimental facilities used to study the $Y$ physics in the last decade (Section 2), we review data related to the coupling of the $n^{3} S$ states to lepton pairs, which include measurements of their masses, leptonic widths $\left(\Gamma_{\text {ee }}\right)$, leptonic branching fractions $\left(\mathbf{B}_{l l}\right)$ and derivation of their total widths (Section 3$)$. Then we turn to a discussion of the radiative transitions to $n^{3} \mathrm{P}$ states (Section 4), which includes measurements of the masses, transition rates, and derivation of their widths. We conclude this part by comparing the measured masses, radiative transition rates, and leptonic widths to the predictions of the potential models (Section 5). Hadronic transitions among the $Y$ states, their branching ratios, and hadronic mass distributions, are the subject of Section 6. In Section 7 we discuss annihilation of $b \bar{b}$ states to gluons. Direct radiative decays of the $Y(1 S)$ are covered in Section 8. We summarize the present experimental status and future prospects in Section 9.

\section{STORAGE RINGS AND DETECTORS}

Except for the initial discovery of the first $Y$ resonances, virtually all other experimental information about the $b \bar{b}$ states is derived from $\mathrm{e}^{+} \mathrm{e}^{-}$storage rings. Two storage rings played a crucial role in revealing more $b \vec{b}$ states and measuring their properties: DORIS at the DESY laboratory in Hamburg, and CESR at Cornell University in Ithaca. Exceptionally precise measurements of the $Y(n S)$ masses were made at the VEPP-4 storage ring in Novosibirsk, which otherwise suffered from lower luminosity $5 \times 10^{30} \mathrm{~cm}^{-2} \mathrm{~s}^{-1}$ (peak value) and a physics program that was unfortunately abbreviated by a fire. DORIS and 
CESR underwent several upgrades over the course of their operations. The DORIS peak luminosity of $3 \times 10^{31} \mathrm{~cm}^{-2} \mathrm{~s}^{-1}$ was matched for the $Y(2 S)$ studies by CESR, but the smaller beam energy spread at CESR enhanced the $Y$ peak cross sections by roughly a factor of two. Since then, the CESR luminosity has been upgraded by an order of magnitude.

There have also been at least two generations of detectors at each facility that were involved in the $Y$ studies. In this article we concentrate only on the more recent experiments. As in the study of atomic spectroscopy, an important role in $Y$ spectroscopy was played by photon detectors. The original CUSB detector at CESR, built of $\mathrm{NaI}(\mathrm{Tl})$ crystals surrounded by lead-glass blocks, provided the first measurements of the radiative transitions from the $Y(3 S)$ and $Y(2 S)(8)$. The Crystal Ball detector, also built of $\mathrm{NaI}(\mathrm{Tl})$ crystals, extensively studied photon transitions in the $\psi$ family (9); it was moved from SPEAR at SLAC to DESY and there it improved the measurements of spectroscopic lines from the $Y(2 S)$ decays. The CUSB detector was later upgraded to CUSB-II by the insertion of higher resolution BGO crystals inside the older NaI array (10). The CUSB, CUSB-II, and Crystal Ball detectors were nonmagnetic. Complementary measurements on $Y$ decays were performed by the magnetic detectors: ARGUS at DORIS (11) and CLEO at CESR (12). Unexpectedly, ARGUS and CLEO played an important role in detection of the $Y(2 S)$ photon lines by employing the low efficiency but high resolution method of detecting photons by conversions to $\mathrm{e}^{+} \mathrm{e}^{-}$pairs. The old CLEO detector was eventually replaced by CLEO-II (13), which was the first (and so far the only one used in this energy range) detector to combine excellent crystal-based photon detection capabilities with excellent charged track reconstruction. The photon detection properties of the above experiments are summarized in Table 1.

\section{COUPLING TO LEPTON PAIRS}

\subsection{Measurement of $Y$ Masses and Their Leptonic Widths}

From the position and height of the $Y$ peaks in $\sigma\left(\mathrm{e}^{+} \mathrm{e}^{-} \rightarrow\right.$ light hadrons) were can extract the $Y$ masses, $M$, and the strength of their coupling to $\mathrm{e}^{+} \mathrm{e}^{-}$, respectively. The latter, expressed in energy units, is called the leptonic width, $\Gamma_{\mathrm{ee}}$. The observed resonance shape is a convolution of the natural resonance shape described by the Breit-Wigner formula, $B W\left(W^{\prime}\right)$, where $W^{\prime}$ is the actual center-of-mass energy of the anni- 
Table 1 Comparison of the photon detectors used to study $Y$ spectroscopy

\begin{tabular}{|c|c|c|c|c|c|c|c|c|}
\hline \multirow[b]{2}{*}{ Detector } & \multirow[b]{2}{*}{ Crystal } & \multirow{2}{*}{$\begin{array}{c}\text { Number of } \\
\text { inner } \\
\text { segments }\end{array}$} & \multirow{2}{*}{$\begin{array}{l}\text { Inner } \\
\text { radius } \\
(\mathrm{cm})\end{array}$} & \multirow{2}{*}{$\begin{array}{c}\% \text { of } \\
4 \pi\end{array}$} & \multicolumn{2}{|c|}{$\begin{array}{c}\sigma_{\mathrm{E}}(\%) \text { at } 100 \\
\mathrm{MeV}\end{array}$} & \multicolumn{2}{|c|}{$\begin{array}{c}\text { Efficiency } \\
(\%)\end{array}$} \\
\hline & & & & & $\gamma \mathrm{X}$ & $\gamma \gamma l^{+} l^{-}$ & $\gamma \mathrm{X}$ & $\gamma \gamma l^{+} l^{-}$ \\
\hline CUSB & $\operatorname{NaI}(T 1)$ & 64 & & 64 & 5.9 & 5.5 & 13.0 & 12 \\
\hline Crystal Ball & NaI(T1) & 672 & 25 & 92 & 4.8 & 4.8 & 15.0 & 22 \\
\hline CUSB-II & BGO & 72 & 8 & 66 & 5.5 & 2.0 & 10.0 & 26 \\
\hline CLEO-II & CsI(T1) & 7800 & 100 & 98 & 4.2 & 3.8 & 50.0 & 25 \\
\hline ARGUS & - & - & - & & 1.1 & - & 0.5 & - \\
\hline CLEO & - & - & - & & 3.4 & - & 2.5 & - \\
\hline
\end{tabular}

a Geometrical acceptance is given as a fraction of the full solid angle $(4 \pi)$. The tabulated efficiency for $\gamma \mathrm{X}$ gives the photon reconstruction efficiency in a multihadronic event, relevant for inclusive spectroscopy. The tabulated efficiency for $\gamma \gamma l^{+} l^{-}$gives the event reconstruction efficiency for events with two photons and two leptons, relevant for detection of photon cascades in the exclusive mode. Photon resolution, $\sigma_{\mathrm{E}}$, is given separately for these two types of measurements. Parameters for the ARGUS and CLEO detectors correspond to photon detection by $\gamma \rightarrow \mathrm{e}^{+} \mathrm{e}^{-}$. CLEO used a lead converter, whereas ARGUS used conversions at the beam-pipe and drift chamber wall.

hilating $\mathrm{e}^{+} \mathrm{e}^{-}$pair, with the probability that the nominal collision energy $W=2 E_{\text {beam }}$ fluctuates to $W^{\prime}, P\left(W, W^{\prime}\right): \mathrm{d} \sigma / \mathrm{d} W=\int \mathrm{d} W^{\prime}$ $B W\left(W^{\prime}\right) P\left(W^{\prime}, W\right)$. The beam energy spread at the storage ring makes $P\left(W, W^{\prime}\right)$ approximately Gaussian, with a rms spread $\Delta W$ typically one or two MeV. Radiation of bremsstrahlung photons by the $\mathrm{e}^{+}$and $\mathrm{e}^{-}$ enhances $P\left(W, W^{\prime}\right)$ for $W^{\prime}<W$, and introduces the high-mass "radiative tail." The shape of $P\left(W, W^{\prime}\right)$ can be calculated to high precision from QED. The Breit-Wigner formula, $B W\left(W^{\prime}\right)=3 \pi \Gamma_{\mathrm{ce}} \Gamma_{\mathrm{had}} /\left\{M^{2}\left[\left(W^{\prime}\right.\right.\right.$ $\left.\left.-M)^{2}+\Gamma_{\text {tot }}^{2} / 4\right]\right\}$, contains the product of the coupling of the resonance to the initial $\left(\Gamma_{\mathrm{ee}}\right)$ and to the final $\left(\Gamma_{\mathrm{had}}\right)$ states. The width of the $B W$ resonance curve depends on the total width of the resonance. Above open-b threshold, the width of the $Y$ resonances $[Y(4 S), Y(5 S), \ldots$ ] is, to a very good approximation, saturated by the decay into b-flavored hadrons: $\Gamma_{\text {tot }}=\Gamma_{\text {had }}=\Gamma_{\mathrm{BB}}$, with a total width of order $10^{4} \mathrm{keV} .{ }^{1}$ In this case, the intrinsic resonance width is larger than the beam energy spread and therefore $\Gamma_{\text {tot }}$ can be measured directly from the observed resonance shape. It should be noted here that the interpretation of the cross-section structure above the $Y(4 \mathrm{~S})$ (see Figure 1) is complicated by the opening of many kinematic thresholds: for $\mathrm{e}^{+} \mathrm{e}^{-} \rightarrow \mathrm{B} \overline{\mathrm{B}}, \mathrm{e}^{+} \mathrm{e}^{-}$ $\rightarrow \mathrm{BB}^{*}, \mathrm{e}^{+} \mathrm{e}^{-} \rightarrow \mathrm{B}_{\mathrm{s}} \overline{\mathrm{B}}_{\mathrm{s}}$, etc. The extracted resonance parameters for $Y(5 S)$ and $Y(6 S)$ are therefore strongly dependent on models used to

\footnotetext{
1 The observation of non-B $\overline{\mathrm{B}}$ decays of the $Y(4 \mathrm{~S})$ by the CLEO collaboration (14) was not supported by more data (15) and seems to have been an upwards fluctuation of the continuum.
} 
describe nonresonant amplitudes (16). The total width for the narrow triplet $S$-state resonances below threshold can be written as a sum of terms, each a partial width of order $1-10 \mathrm{keV}$ :

$$
\Gamma_{\mathrm{tot}}=\Gamma_{\mathrm{ggg}}+\Gamma_{\mathrm{gg} \gamma}+R \Gamma_{\mathrm{ee}}+3 \Gamma_{\mathrm{ee}}+\Gamma_{\gamma}+\Gamma_{\pi \pi},
$$

These partial widths express the decays into three gluons (ggg), two gluons and a photon (gg $\gamma$ ), $\mathrm{q} \overline{\mathrm{q}}$, dileptons, parity-changing electromagnetic radiative decays (if allowed), and parity-conserving dipion decays (if allowed), respectively; the value $R$ is the ratio of inclusive production of hadrons to the mu-pair production rate at $W=M_{Y}$. For the Pstate resonances, only the first term and the last two terms are present; but the first term must be replaced by $\Gamma_{\mathrm{gg}}$ for the spin- 0 and spin- 2 states, or $\Gamma_{\mathrm{q} a \mathrm{~g}}$ for the spin-1 state (17).

For the $Y$ resonances below the open flavor threshold, which are produced directly in $\mathrm{e}^{+} \mathrm{e}^{-}$annihilations, $\Gamma_{\text {tot }}$ is negligibly small compared with $\Delta W$, and the natural line shape cannot be mapped directly. In this regime, the Breit-Wigner function can be approximated by a delta function at the resonance mass: $B W\left(W^{\prime}\right) \approx \delta\left(W^{\prime}-M\right)$ $6 \pi^{2} \Gamma_{\text {ee }} \Gamma_{\text {hacl }} /\left(M^{2} \Gamma_{\text {tot }}\right)$. Since hadronic decays dominate the $Y$ decays, one obtains $\Gamma_{\text {tot }} \approx \Gamma_{\text {had }}$, and $\tilde{\Gamma}_{\text {ee }} \equiv \Gamma_{\mathrm{ee}} \Gamma_{\mathrm{had}} / \Gamma_{\text {tot }} \approx \Gamma_{\mathrm{ee}}$. From a fit to the resonant enhancement in the observed hadrionic cross section (see Figure 1) we can determine $M$ and $\tilde{\Gamma}_{\text {ee }}(18-21)$.

When calculated from the storage-ring magnet currents, the absolute beam energy scale is uncertain to about $10-30 \mathrm{MeV}$, and this uncertainty contributes to the systematic error of the mass measurements. Statistical errors on $M$ in a typical measurement are a factor of 10 to 100 better. The systematic error can be reduced to the level of statistical uncertainty by the beam depolarization technique pioneered at Novosibirsk (22), and later applied at DORIS and CESR, yielding an impressive accuracy of $\Delta M / M \sim 5 \times 10^{-5}$ for the $Y$ mass measurements (23).

\subsection{Measurement of Leptonic Branching Ratios}

The $Y$ states can decay to lepton pairs $\mathrm{e}^{+} \mathrm{e}^{-}, \mu^{+} \mu^{-}$, and $\tau^{+} \tau^{-}$via a virtual photon. Since coupling of the virtual photon to leptons depends only on their electric charges and not the flavor, the branching ratios for these processes are expected to be equal, $B_{\mathrm{ee}}=B_{\mu \mu}=B_{\tau \tau} \equiv B_{l l}$ (neglecting small phase space differences). By definition, $B_{l l}=\Gamma_{l l} / \Gamma_{\text {tot }}$. Experimentally we measure $\tilde{B}_{l l}=\Gamma_{l l} / \Gamma_{\text {had }}$. Because $\Gamma_{\text {had }} \approx \Gamma_{\text {tot }}$, we find $\bar{B}_{l l} \approx B_{l l}$ and $B_{l l} \ll 1$. Assuming that all leptonic rates are equal, and assuming that the sum of the hadronic width and leptonic widths saturate the total width, we have $\Gamma_{\text {tot }}=\Gamma_{\text {had }}+3 \Gamma_{l l}$, from which $B_{l l}$ 


\section{2}

\section{BESSON \& SKWARNICKI}

$=\Gamma_{l l} /\left(\Gamma_{\text {had }}+3 \Gamma_{l l}\right)=\tilde{B}_{l l} /\left(1+3 \tilde{B}_{l l}\right)$. Lepton pair and hadronic production rates are measured on and off the resonance peak to subtract nonresonant backgrounds in the $\tilde{B}_{l l}$ determination. It was recently pointed out that, for data taken on the resonance but off its peak value, interference effects between the resonant and continuum lepton pair production processes can be non-negligible (24). The effect of the continuum, in particular, the large continuum subtraction, dominates the measurement errors. The large continuum $\mathrm{e}^{+} \mathrm{e}^{-} \rightarrow \mathrm{e}^{+} \mathrm{e}^{-}$cross section makes this method impractical for $B_{\text {ee }}$; thus $B_{\mu \mu}$ is the preferred way of measuring $B_{l l}(20,21,24,25)$. To test universality of lepton couplings, CLEO and ARGUS measured $B_{\tau \tau}$ for $Y(1 S)$ and $Y(2 S)(21,26)$. Within errors, the values agreed with $B_{\mu \mu}$. For the $Y(1 S)$, ARGUS and CLEO (27) also measured $B_{\mu \mu}$ and $B_{\text {ee }}$ by dividing the measured rate for $Y(2 S)$ $\rightarrow \pi^{+} \pi^{-} Y(1 S) \rightarrow \pi^{+} \pi^{-} l^{+} l^{-}$by the rate observed for $Y(2 S) \rightarrow$ $\pi^{+} \pi^{-} Y(1 S) \rightarrow \pi^{+} \pi^{-}$hadrons (see Section 6 for a discussion of hadronic transitions). This method does not require any continuum subtraction.

\subsection{Derivation of the Total Widths of the $Y$ Resonances}

With the experimental value of $B_{l}$ we can unfold $\tilde{\Gamma}_{\mathrm{ee}}$ to determine the true value of $\Gamma_{\mathrm{ee}}=\tilde{\Gamma}_{\mathrm{ee}} /\left(1-3 B_{l l}\right)$. From $\Gamma_{\mathrm{tot}}=\Gamma_{\mathrm{ee}} / B_{\mathrm{ee}} \equiv \Gamma_{\mathrm{ee}} / B_{l l}$, we can derive experimental values for the total widths of the $Y$ states. In the above equation, we cancel $\Gamma_{\mathrm{ee}}$ derived from the formation process, $\mathrm{e}^{+} \mathrm{e}^{-} \rightarrow Y$, with the $\Gamma_{\mathrm{ec}}$ defined for the decay, $Y \rightarrow \mathrm{e}^{+} \mathrm{e}^{-}$. Therefore, it is important to use in the $P\left(W^{\prime}, W\right)$ calculations only those radiative corrections that do not contribute to the decay process and let the other radiative processes be absorbed into the definition of $\Gamma_{\text {ee }}(18,28)$. This distinction was overlooked in early total width derivations, which resulted in an underestimation of the total widths by about $7 \%$. Parameters derived from couplings of the $Y(n S)$ states to lepton pairs are summarized in Table 2.

\subsection{Comparison with Theoretical Predictions}

For a quarkonium resonance, we expect $\Gamma_{\text {ce }}$ to be proportional ( $a$ ) to the square of the quark charge, $e_{\mathrm{b}}^{2}\left(e_{\mathrm{b}}=-1 / 3\right) ;(b)$ to the electromagnetic coupling constant, $\alpha ;(c)$ to the probability of having the quark-antiquark pair at the production (annihilation) point expressed by the square of the wave function at the origin, $|\Psi(0)|^{2}$; and $(d)$ to the energy dependence of the photon propagator, $1 / M^{2}$. This dependence is reflected in the Van Royen-Weisskopf (29) expression: $\Gamma_{\mathrm{ee}}$ $=16 \pi \alpha e_{\mathrm{b}}^{2}|\Psi(0)|^{2} / M^{2}$. The first-order QCD correction has been de- 
Table 2 Parameters (these and other data on the resonances are taken from Ref. 89) for $n^{3} S_{1} b \bar{b}$ states measured from their coupling to lepton pairs

\begin{tabular}{crlcc}
\hline \hline Resonance & \multicolumn{1}{c}{$M(\mathrm{MeV})$} & $B_{l l}(\%)$ & $\Gamma_{\text {ee }}(\mathrm{keV})$ & $\Gamma_{\text {tot }}(\mathrm{keV})$ \\
\hline 1S & $9460.3 \pm 0.2$ & $2.5 \pm 0.06$ & $1.34 \pm 0.04$ & $52.1 \pm 2.1$ \\
2S & $10023.3 \pm 0.3$ & $1.3 \pm 0.2$ & $0.59 \pm 0.03$ & $43.0 \pm 8.0$ \\
3S & $10355.3 \pm 0.5$ & $1.8 \pm 0.2$ & $0.44 \pm 0.03$ & $24.3 \pm 2.9$ \\
4S & $10580.0 \pm 3.5$ & & $0.24 \pm 0.05$ & $23.8 \pm 2.210^{3}$ \\
\hline
\end{tabular}

termined to be $1-16 \alpha_{s} / 3 \pi$, which may be as large as $40 \%$, depending on the choice for the value of the strong coupling constant $\alpha_{\mathrm{s}}$ (30). Most of the potential models discussed in Section 5 can predict values for $|\Psi(0)|^{2}$ in rough agreement with the measured $\Gamma_{\mathrm{ee}}$ values.

The probability for getting both quarks together, $|\Psi(0)|^{2}$, will enter any formula for annihilation of the $n \mathrm{~S}$ states. For example, annihilation into three gluons can be expressed (31) in lowest QCD order by $\Gamma_{\mathrm{ggg}}$ $=160\left(\pi^{2}-9\right) \alpha_{s}^{3}|\Psi(0)|^{2} /\left(81 M^{2}\right)$. Instead of using a model-dependent $|\Psi(0)|^{2}$, it is customary to normalize any annihilation rate to $\Gamma_{\mathrm{ee}}$. In the ratio, $|\Psi(0)|^{2}$ drops out, leaving only perturbative terms. The experimental value of $\Gamma_{\mathrm{ggg}}$ can be obtained by subtracting from $\Gamma_{\text {tot }}$ contributions from single-photon annihilation rates to lepton and quark pairs (and $\mathrm{b} \overline{\mathrm{b}}$ transition rates for excited $n \mathrm{~S}$ states, $n \geq 2$ ). Unfortunately, the theoretical uncertainties in higher order QCD corrections to $\Gamma_{\mathrm{ggg}}$ are large (32), which limits the usefulness of the $\Gamma_{\mathrm{ggg}} / \Gamma_{\mathrm{ee}}$ ratio for determining $\alpha_{\mathrm{s}}$. A better method to determine $\alpha_{\mathrm{s}}$ from $Y$ decays is discussed in Section 8.1.

\section{MEASUREMENTS OF RADIATIVE TRANSITIONS}

Because of the large $b$ quark mass, the $b \bar{b}$ states are the most compact bound states known in nature: $\bar{R}^{2} 1 / 2 \approx 0.2 \mathrm{fm}$ for the $Y(1 \mathrm{~S})$. Photon wavelengths in the radiative transitions among the $b \bar{b}$ states are larger than or at most comparable to the sizes of the radiating system; thus dipole transitions dominate. From conservation of $P$ and $C$ parities it follows that electric dipole transitions (E1) can occur between states with $\Delta S=0$ and $\Delta L= \pm 1$, whereas magnetic dipole transitions (M1) can occur between states with $\Delta S= \pm 1$ and $\Delta L=0$. Thus, only M1 transitions can connect triplet $(S=1)$ and singlet $(S=0)$ sectors. M1 transitions are expected to be rare compared to $\mathrm{E} 1$ transitions because of the heavy b-quark mass. 


\subsection{Radiative Transitions from the $Y(2 S)$}

Applied to the $n^{3} S_{1--}$ states, the above selection rules imply that the radiative decays of these states will be predominantly to the $n^{\prime 3} \mathrm{P}_{J^{++}}$ states $(J=0,1,2)$, also called $\chi_{\mathrm{b}}$ states. The E1 transitions from the $Y(2 S)$ state, for instance, lead to the $\chi_{\mathrm{b}}(1 \mathrm{P})$ states. These transitions were observed in four experiments-CUSB (34), CLEO (35), Crystal Ball (36), and ARGUS (37) - by measuring the energy distribution of transition photons detected "inclusively" in multihadronic events: $Y(2 S) \rightarrow \gamma \chi_{\mathrm{b}} ; \chi_{\mathrm{b}} \rightarrow$ hadrons. Three closely spaced spectroscopic lines were observed corresponding to the transitions to the $J=2,1,0 \chi_{\mathrm{b}}(1 \mathrm{P})$ states (see Figure 3 ). The widths of these lines are consistent with the experimental energy resolution, which indicates that the $\chi_{\mathrm{b}}$ states are also long-lived objects. Measurement of the photon energies in these transitions determines the $\chi_{b}$ masses and the amplitude of each line determines $B\left(2 \mathrm{~S}_{1} \rightarrow \gamma 1 \mathrm{P}_{J}\right)$. The fourth, high energy line observed in the inclusive photon spectrum shown in Figure 3 corresponds to the subsequent E1 transition from the $\chi_{\mathrm{b}}(1 \mathrm{P})$ states to the $Y(1 \mathrm{~S})$, followed by its hadronic decay. Transitions from the different $J$ states to the lower mass $\mathrm{S}$ state fall in the region where the experimental resolution is too poor to discern the small fine structure of the $\chi_{b}$ states.

In the above inclusive measurement method, the radiative transition lines sit on a very large photon background coming from $\pi^{0}$ decays. Furthermore, continuum hadronic events, $\mathrm{e}^{+} \mathrm{e}^{-} \rightarrow \mathrm{q} \overline{\mathrm{q}}$, cannot be dis-

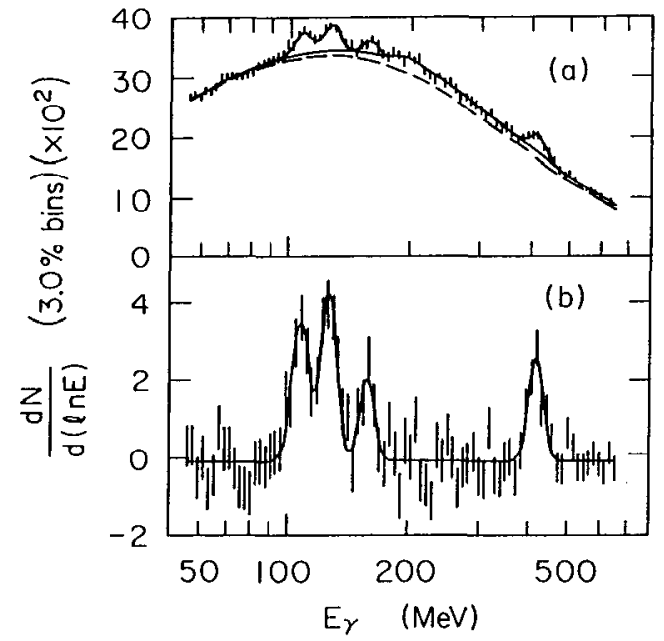

Figure 3 (a) Inclusive photon energy spectrum, data taken on the $\mathrm{Y}(2 \mathrm{~S})$ resonance, from the Crystal Ball collaboration (36). (b) Background subtracted spectrum. 
tinguished from $\mathrm{e}^{+} \mathrm{e}^{-} \rightarrow \mathrm{b} \overline{\mathrm{b}}$ on an event-by-event basis; thus both contribute to the photon background. The radiative cascade transitions, $Y(2 S) \rightarrow \gamma \chi_{\mathrm{b}}(1 \mathrm{P}), \chi_{\mathrm{b}}(1 \mathrm{P}) \rightarrow \gamma Y(1 \mathrm{~S})$, can also be detected in the "exclusive" mode by tagging the $Y(1 S)$ state by its decay to the lepton pairs $\mathrm{e}^{+} \mathrm{e}^{-}$or $\mu^{+} \mu^{-}$(see, for example, Figure 4, discussed later in the text); hadronic event background is thereby eliminated. The remaining photon background is small and comes mostly from doubly radiative $\mathrm{e}^{+} \mathrm{e}^{-}$annihilation into $\mathrm{e}^{+} \mathrm{e}^{-}$or $\mu^{+} \mu^{-}$(the $\mathrm{e}^{+} \mathrm{e}^{-}$channel has a bigger background). The observed signal statistics are unfortunately also small, primarily because of the small $B_{l l}$ for the $Y$ states. Nevertheless, these measurements are very important because they are sensitive to the product branching ratio, $B\left(2 \mathrm{~S}_{1} \rightarrow \gamma 1 \mathrm{P}_{J}\right) B\left(1 \mathrm{P}_{J} \rightarrow\right.$ $\gamma 1 S_{1}$ ), where the $J$ value is determined by the energy of the lower energy photon in the cascade. Dividing this product branching ratio by the inclusively measured $B\left(2 \mathrm{~S}_{1} \rightarrow \gamma 1 \mathrm{P}_{J}\right)$, we can extract $B\left(1 \mathrm{P}_{J} \rightarrow \gamma 1 \mathrm{~S}_{1}\right)$. The radiative cascades from $Y(2 S)$ to $Y(1 S)$ via the $\chi_{\mathrm{b}}(1 \mathrm{P})$ states were measured by the CUSB (38) and the Crystal Ball (39) experiments. The $J=0$ state has not been detected in the radiative cascade transitions, which indicates that the gluonic decay width of this state dominates over the radiative width. The Crystal Ball experiment also analyzed angular correlations among the photons and leptons in the above cas-

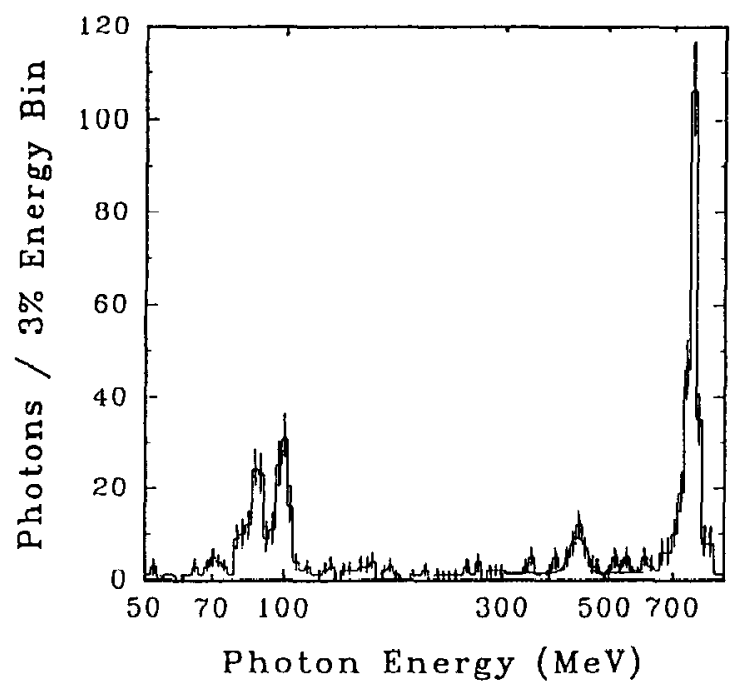

Figure 4 CUSB photon energy spectrum for events consistent with $Y(3 S) \rightarrow \gamma \gamma Y$, where the daughter $Y$ decays into $\mu^{+} \mu^{-}$, or $\mathrm{e}^{+} \mathrm{e}^{-}$. The peak around $450 \mathrm{MeV}$ corresponds to the $\gamma(3 S) \rightarrow \gamma \chi_{b}(1 \mathrm{P}) \rightarrow \gamma \gamma \gamma(1 S)$ transitions (two entries per event). 
cade transition and determined the spin of the $\chi_{b}\left(1^{3} \mathrm{P}_{J}\right)$ states in a model-independent way (40). The multipole analysis showed that E1 transitions were preferred by the data, but the statistical error did not exclude the possibility of small M2 admixtures (41).

\subsection{Radiative Transitions from the $Y(3 S)$}

Radiative transitions from the $Y(3 S)$ are more complex. Electric dipole transitions may produce the $\chi_{\mathrm{b}}(2 \mathrm{P})$ or the $\chi_{\mathrm{b}}(1 \mathrm{P})$ states. Furthermore, the $\chi_{\mathrm{b}}(2 \mathrm{P})$ states may decay to the $Y(2 \mathrm{~S}), Y(1 \mathrm{~S})$, or $Y(1 \mathrm{D})$ states. The $Y(1 D)$ states may in turn decay to the $\chi_{b}(I P)$ states. Recently, a high statistics, high resolution inclusive study of $\gamma(3 S) \rightarrow \gamma \chi_{\mathrm{b}}(2 \mathrm{P})$ was published by the CLEO-II experiment, improving on the earlier CUSB (43) and CUSB-II (44) measurements (see Figure 5). The fine segmentation of the CLEO-II calorimeter (see Table 1) offered no advantage, however, over the coarsely segmented CUSB-II detector for the low multiplicity exclusive studies $(45,46)$. The photon spectrum for $\gamma \gamma l^{+} l^{-}$ events from the CUSB-II study is shown in Figure 4. The transitions $Y(3 S) \rightarrow \gamma \chi_{b}(2 \mathrm{P}), \chi_{\mathrm{b}}(2 \mathrm{P}) \rightarrow \gamma Y(2 \mathrm{~S})$, and $\chi_{\mathrm{b}}(2 \mathrm{P}) \rightarrow \gamma \gamma(1 \mathrm{~S})$ were observed by both experiments. CUSB-II presented evidence for the rare $Y(3 S) \rightarrow \gamma \chi_{\mathrm{b}}(1 \mathrm{P})$ decays in the exclusive and the inclusive analyses, $B(3 S \rightarrow \gamma 1 \mathrm{P}) B(1 \mathrm{P} \rightarrow \gamma 1 \mathrm{~S})=(1.6 \pm 0.4) \times 10^{-3}$, but the individual photon lines for different spins of the $\chi_{\mathrm{b}}(1 \mathrm{P})$ were not resolved. The transitions of the $Y(1 D)$ states have not yet been observed. They are hidden in the inclusive photon spectrum under more intense spectroscopic lines from transitions between the $S$ and $P$ states. This problem can be avoided in the exclusive analysis; however, the available data samples are lacking sufficient statistical power to detect the expected (75) but rare quadruple radiative cascades $Y(3 \mathrm{~S}) \rightarrow \gamma \chi_{\mathrm{b}}(2 \mathrm{P}) \rightarrow \gamma \gamma \gamma(1 \mathrm{D})$ $\rightarrow \gamma \gamma \gamma \chi_{\mathrm{b}}(1 \mathrm{P}) \rightarrow \gamma \gamma \gamma \gamma \gamma(1 \mathrm{~S}) \rightarrow \gamma \gamma \gamma \gamma l^{+} l^{-}$. There is also no evidence for direct $n^{3} \mathrm{~S}_{1^{--}} \rightarrow \gamma n^{1} \mathrm{~S}_{0^{-+}}$or hindered $n^{3} \mathrm{~S}_{1^{--}} \rightarrow \gamma n^{\prime 1} \mathrm{~S}_{0^{-+}} \mathrm{M} 1$ transitions in the $Y$ system, which have already been observed in $\mathrm{c} \overline{\mathbf{c}}$ quarkonium (47). The heavier b quark mass suppresses the rates for these transitions and the experimental searches suffer from increased $\pi^{0}$ background.

Results for radiative transitions between the triplet $S$ and the triplet $P$ states are summarized ${ }^{2}$ in Tables 3 and 4.

\footnotetext{
${ }^{2}$ The branching ratios calculated here for $2 S \rightarrow \gamma 1 \mathrm{P}$ transitions differ slightly from those calculated by the Particle Data Group (PDG) (89) because the PDG neglects systematic errors on the CUSB results, whereas the CUSB paper (34) gives systematic errors larger than the statistical ones. PDG also neglects the systematic errors in the energy determination of the $3 \mathrm{~S} \rightarrow \gamma 2 \mathrm{P}$ photon energies from the CUSB-II experiment. Many derived quantities in the tables included here and quoted in the later sections may have
} 


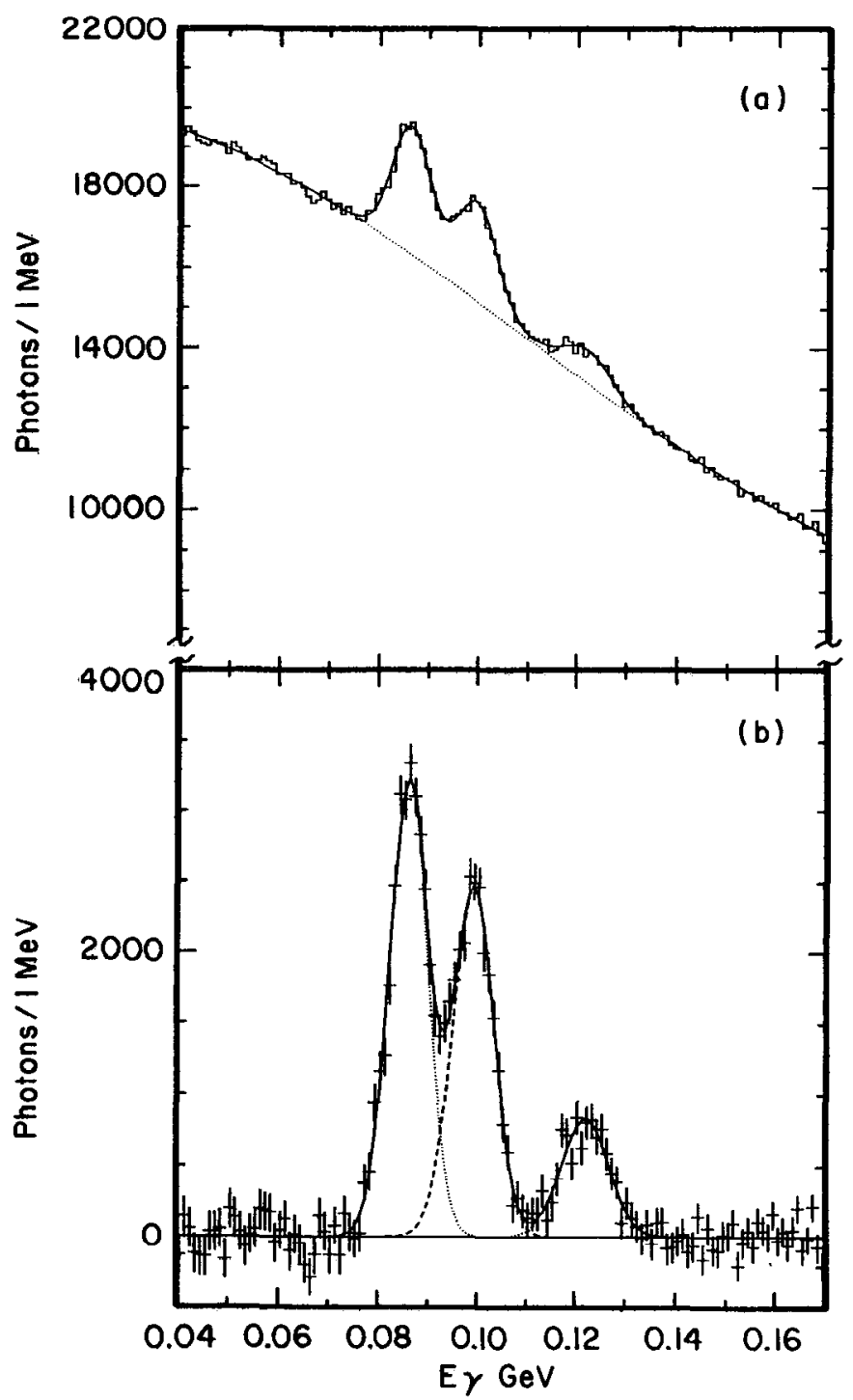

Figure 5 (a) Inclusive $Y(3 S)$ photon energy spectrum from the CLEO-II collaboration. (b) Background subtracted spectrum.

errors not directly calculable from the ones listed for the contributing measurements as we have tried to keep (cancel out) the systematic errors of the experiments in the average values (in the ratios). We have also corrected all results for the most recent values of $B_{l l}$ and masse:s of the 1S, 2S, and $3 S$ states. 
Table 3 Parameters of the radiative transitions from $S$ states to the $P$ states

\begin{tabular}{lcrrrrr}
\hline \multirow{2}{*}{ Transition } & $J^{\mathrm{a}}$ & \multicolumn{1}{c}{$B(\%)$} & \multicolumn{1}{c}{$E_{\gamma}(\mathrm{MeV})$} & \multicolumn{1}{c}{$M^{\mathrm{a}}(\mathrm{MeV})$} & $M_{\mathrm{cog}}(\mathrm{MeV})$ & $\frac{M_{J=2}-M_{J=1}}{M_{J=1}-M_{J=0}}$ \\
\hline \multirow{3}{*}{$2 \mathrm{~S} \rightarrow \gamma 1 \mathrm{P}$} & 1 & $7.0 \pm 1.0$ & $130.4 \pm 0.7$ & $9892.1 \pm 0.7$ & $9900.2 \pm 0.7$ & $0.65 \pm 0.03$ \\
& 0 & $4.2 \pm 0.9$ & $162.3 \pm 1.3$ & $9859.7 \pm 0.6$ & & \\
& 2 & $11.4 \pm 0.6$ & $86.5 \pm 0.4$ & $10268.5 \pm 0.6$ & & \\
$3 \mathrm{SS} \rightarrow \gamma 2 \mathrm{P}$ & 1 & $11.5 \pm 0.6$ & $99.7 \pm 0.4$ & $10255.2 \pm 0.7$ & $10260.0 \pm 0.7$ & $0.576 \pm 0.014$ \\
& 0 & $5.4 \pm 0.5$ & $122.4 \pm 0.6$ & $10232.1 \pm 0.8$ & &
\end{tabular}

a $J$ denotes spin of the $P$ state, and $M$ is its mass. The $3 S$ data are taken from References $42,44,45,46$. The $2 S$ data are taken from References 34-40. See also footnote 2 .

\section{COMPARISON WITH POTENTIAL MODELS}

In the nonrelativistic limit, quark dynamics should be well described by a static potential. The form of the interquark potential has been the subject of phenomenological modeling and calculation based on the theory of strong interactions, quantum chromodynamics. In this section we compare the experimental data with the predictions of these models.

\subsection{Excitation Energies}

A primary test of a potential model is how well its predictions compare with the observed excitation energies of various bound states, that is, the masses of the $b \bar{b}$ levels. In the strict nonrelativistic approach, there are no spin-dependent forces. Since spin-dependent shifts are observed in the data, mass predictions of the static potential must be compared with spin-averaged measurements, i.e. so-called center-of-gravity masses: $M_{\text {cog }}=\sum_{J}(2 J+1) M_{J} / \sum_{J}(2 J+1)$. Masses have been measured for five triplet states below $\mathrm{BB}$ threshold: 1S, 2S, 3S, 1P, 2P (see Tables 2 and 3). We have calculated a mean square deviation between

Table 4 Branching ratios measured from two photon cascade transitions

\begin{tabular}{|c|c|c|c|c|}
\hline & \multirow{2}{*}{$\begin{array}{l}\text { P-state } \\
\text { spin }\end{array}$} & \multicolumn{3}{|c|}{$P \rightarrow S^{\prime}$} \\
\hline & & $1 \mathrm{P} \rightarrow \gamma 1 \mathrm{~S}$ & $2 \mathrm{P} \rightarrow \gamma 2 \mathrm{~S}$ & $2 \mathrm{P} \rightarrow y 1 \mathrm{~S}$ \\
\hline $\begin{array}{c}B(\mathrm{~S} \rightarrow \mathrm{P}) B(\mathrm{P} \rightarrow \mathrm{S}) B\left(\mathrm{~S} \rightarrow l^{+} l^{-}\right) \\
\left(10^{-4}\right)\end{array}$ & $\begin{array}{l}2 \\
1 \\
0\end{array}$ & $\begin{array}{c}4.0 \pm 0.8 \\
6.1 \pm 0.9 \\
<0.5 \text { (90\% C.L.) }\end{array}$ & $\begin{array}{l}5.4 \pm 0.6 \\
7.4 \pm 0.7 \\
0.8 \pm 0.4\end{array}$ & $\begin{array}{l}4.2 \pm 0.5 \\
5.0 \pm 0.6 \\
0.3 \pm 0.2\end{array}$ \\
\hline$B(\mathrm{P} \rightarrow \mathrm{S})$ & $\begin{array}{l}2 \\
1 \\
0\end{array}$ & $\begin{array}{c}24 \pm 8 \\
35 \pm 11 \\
<5(90 \% \text { C.L. })\end{array}$ & $\begin{array}{l}36 \pm 7 \\
49 \pm 10 \\
12 \pm 6\end{array}$ & $\begin{array}{r}15 \pm 4 \\
17 \pm 4 \\
2 \pm 2\end{array}$ \\
\hline
\end{tabular}


the theoretical predicted $\left(M_{\mathrm{th}}\right)$ and the measured masses ${ }^{3}\left(M_{\mathrm{m}}\right)$ : $\sigma^{2}(\Delta M)=(\Delta M-\overline{\Delta M})^{2}, \Delta M=M_{\mathrm{th}}-M_{\mathrm{m}}$, for various potential models. This procedure has the virtue of being insensitive to an overall shift between the theoretical and the measured mass scales; thus allowing comparisons with those models developed before the absolute mass scale was well determined experimentally.

The nonrelativistic potential models can also be tested against the measured values of the $\Gamma_{\mathrm{ee}}$ 's of the $Y(n S)$ states (see Section 3.4). Theoretical and experimental uncertainties are reduced when the ratio of $\Gamma_{\mathrm{ee}}$ widths is calculated: $\mathscr{R}_{\mathrm{ee}}(n \mathrm{~S})=\Gamma_{\mathrm{ee}}(n \mathrm{~S}) / \Gamma_{\mathrm{ee}}(1 \mathrm{~S})$. We have calculated the relative accuracy of the theoretical predictions, $\delta \mathscr{R}_{\mathrm{ee}} / \mathscr{R}_{\mathrm{ee}}$ $=\left(\mathscr{R}_{\mathrm{ec}}^{\mathrm{th}}-\mathscr{R}_{\mathrm{ee}}^{\mathrm{m}}\right) / \mathscr{R}_{\mathrm{ec}}^{\mathrm{m}}$, by calculating the weighted average over the two measured ratios $(2 S / 1 S$ and $3 S / 1 S)$, where the input weights correspond to the varying levels of precision of the available experimental data. Potential models also predict the electric dipole transitions widths, $\Gamma_{\mathrm{E} 1}$ (see Section 5.2). Again, we have calculated, using the experimental errors as weights, the relative accuracy of these predictions, $\delta \Gamma_{\mathrm{E} 1} / \Gamma_{\mathrm{E} 1}$, using the measured transition widths for $2 \mathrm{~S} \rightarrow \gamma 1 \mathrm{P}$, $3 \mathrm{~S} \rightarrow \gamma 2 \mathrm{P}$ and the ratio of the transition widths $(2 \mathrm{P} \rightarrow \gamma 1 \mathrm{~S}) /(2 \mathrm{P} \rightarrow \gamma 2 \mathrm{~S})$. Some of the potential models employ relativistic formalism or apply relativistic corrections to the nonrelativistic predictions. These models predict the magnitude of the spin-dependent splitting of the $1 \mathrm{P}$ and $2 \mathrm{P}$ states, discussed at some length below. Here, for the relativistic models we calculated a mean square deviation between the predicted and measured mass splitting: $\sigma^{2}\left(\delta M_{J}\right)=\overline{\left[\left(M_{J}-M_{J-1}\right)_{\mathrm{th}}\right.}-$ $\left.\left(M_{J}-M_{J-1}\right)_{\mathrm{m}}\right]^{2}$, where $J=1,2$, neglecting the experimental errors in this case.

Table 5 shows $\sigma(\Delta M), \delta \mathscr{R}_{\mathrm{ee}} / \mathscr{R}_{\mathrm{ee}}, \delta \Gamma_{\mathrm{E} 1} / \Gamma_{\mathrm{E} 1}$, and $\sigma\left(\delta M_{J}\right)$ for a number of the potential models. The dominant dependence of the potential on the interquark separation $(R)$ at short $(R<0.1 \mathrm{fm})$ and long $(R>1.0$ $\mathrm{fm})$ distances is indicated. The symbols used in the table are explained below. Since the precision of the theoretical predictions may depend on the quality of the input data used in adjusting the free parameters of the model, we have included the submission date of each publication considered. The number of free parameters $\left(N_{\mathrm{P}}\right)$ in the model, excluding the $b$ quark mass, is also displayed in the table. We indicate whether the input data used to fix the free parameters came from the $\mathrm{b} \overline{\mathrm{b}}$ or $c \overline{\mathrm{c}}$ systems. The models are ordered according to the best spinaveraged mass predictions.

\footnotetext{
${ }^{3}$ Experimental errors on the mass measurements are small compared to theoretical uncertainties.
} 
Table 5 Comparison of potential model predictions with $b \bar{b}$ data

\begin{tabular}{|c|c|c|c|c|c|c|c|c|c|c|c|}
\hline \multirow{2}{*}{$\begin{array}{l}\text { Model } \\
\text { number }\end{array}$} & \multirow[b]{2}{*}{ Author(s) } & \multirow[b]{2}{*}{ Year } & \multicolumn{2}{|c|}{ Potential } & \multirow[b]{2}{*}{$N_{\mathbf{P}}$} & \multirow{2}{*}{$\begin{array}{l}\text { Input } \\
\text { data }\end{array}$} & \multirow{2}{*}{$\begin{array}{c}\sigma \\
\Delta M \\
(\mathrm{MeV})\end{array}$} & \multirow{2}{*}{$\begin{array}{c}\Delta M \\
4 \mathrm{~S} \\
(\mathrm{MeV})\end{array}$} & \multirow{2}{*}{$\begin{array}{c}\frac{\delta \mathscr{R}_{\mathrm{ee}}}{\mathscr{R}_{\mathrm{cc}}} \\
(\%)\end{array}$} & \multirow{2}{*}{$\begin{array}{l}\frac{\delta \Gamma_{\mathrm{E} 1}}{\Delta_{\mathrm{EI}}} \\
(\%)\end{array}$} & \multirow{2}{*}{$\begin{array}{c}\sigma \\
\delta M_{\mathrm{J}} \\
(\mathrm{MeV})\end{array}$} \\
\hline & & & short & long & & & & & & & \\
\hline 1 & Fulcher (48) & 89 & $\mathrm{Cl}$ & $R$ & 1 & $\mathrm{~b} \overline{\bar{b}}$ & 2.3 & & 5 & 15 & 2.8 \\
\hline 2 & Lichtenberg et al (49) & 88 & $\mathrm{C} 1$ & $R$ & 2 & $\mathrm{~b} \overline{\mathrm{b}}$ & 2.7 & +33 & & & \\
\hline 3 & Lichtenberg et al (49) & 88 & $R^{-0.75}$ & $R^{+0.75}$ & 3 & $\mathrm{~b} \overline{\mathrm{b}}$ & 2.8 & +33 & & & \\
\hline 4 & Fulcher $(50)$ & 88 & $\mathrm{C} 3$ & $\boldsymbol{R}$ & 4 & $\mathrm{~b} \overline{\mathrm{b}}$ & 3.5 & & & & \\
\hline 5 & Fulcher (51) & 91 & $\mathrm{C} 1$ & $R$ & 2 & $\mathrm{~b} \bar{b}$ & 3.8 & & 1 & & 3.3 \\
\hline 6 & Gupta et al (52) & 87 & $\mathrm{C} 1$ & $R$ & 3 & $\mathbf{b} \bar{b}$ & 3.9 & & 4 & & 1.7 \\
\hline 7 & Buchmüller et al (53) & 80 & $\mathrm{C} 2$ & $R$ & 2 & $\underline{\mathrm{b}} \overline{\mathrm{b}}$ & 4.3 & +46 & 2 & 13 & \\
\hline 8 & Moxhay \& Rosner (54) & 83 & $\mathrm{C} 1$ & $R$ & 3 & $\mathrm{~b} \overline{\mathrm{b}}, \mathrm{c} \overline{\mathrm{c}}$ & 4.3 & +28 & 8 & 15 & \\
\hline 9 & Richardson (55) & 78 & $\mathrm{C} 1$ & $R$ & 1 & $c \overline{\mathbf{c}}$ & 4.3 & +33 & 3 & & \\
\hline 10 & Gupta et al (56) & 82 & $\mathrm{C} 3$ & $R$ & 3 & $\mathrm{~b} \overline{\mathrm{b}}, \mathrm{c} \overline{\mathrm{c}}$ & 4.7 & & 10 & 15 & 4.6 \\
\hline 11 & Bhanot \& Rudaz (58) & 78 & $\mathrm{Co}$ & $R$ & 3 & $\mathbf{c} \overline{\mathbf{c}}$ & 6.8 & & 2 & & \\
\hline 12 & Lichtenberg et al (49) & 88 & $R^{-0.5}$ & $R^{+0.5}$ & 3 & $\mathrm{~b} \overline{\bar{b}}$ & 7.3 & +22 & & & \\
\hline 13 & Ito $(59)$ & 89 & $\mathrm{Co}$ & $R$ & 5 & $\mathrm{~b} \overline{\mathbf{b}}$ & 7.5 & & 6 & & \\
\hline 14 & Lichtenberg et al (49) & 88 & $\mathrm{CO}$ & $R$ & 3 & $\mathrm{~b} \overline{\mathrm{b}}$ & 8.9 & +46 & & & \\
\hline 15 & Bander et al (60) & 83 & $\mathrm{C} 1$ & $R$ & 3 & $\mathrm{~b} \overline{\mathrm{b}}, \mathrm{c} \overline{\mathrm{c}}$ & 9.1 & +9 & 9 & 36 & 4.0 \\
\hline 16 & Quigg \& Rosner (62) & 79 & \multicolumn{2}{|c|}{$\log R$} & 2 & $\mathbf{c} \overline{\mathbf{c}}$ & 10.9 & +10 & 7 & & \\
\hline 17 & Eichten \& Feinstein (63) & 80 & $\mathrm{CO}$ & $R$ & 2 & $\mathrm{~b} \overline{\mathrm{b}}, \mathrm{c} \overline{\mathrm{c}}$ & 11.1 & & & & 5.0 \\
\hline 18 & Krasemann \& Ono (64) & 79 & $\mathrm{C} 1$ & $R$ & 4 & $\mathrm{~b} \overline{\mathrm{b}}, \mathrm{c} \overline{\mathrm{c}}$ & 11.3 & +7 & 4 & & \\
\hline 19 & McClary \& Byers (65) & 83 & $\mathrm{CO}$ & $R$ & 4 & $\mathrm{~b} \overline{\mathrm{b}}, \mathrm{c} \overrightarrow{\mathrm{c}}$ & 11.6 & +33 & & 20 & 12.2 \\
\hline 20 & Eichten et al (60) & 79 & $\mathrm{CO}$ & $R$ & 2 & $\mathrm{c} \overline{\mathbf{c}}$ & 13.3 & +48 & 20 & 15 & \\
\hline 21 & Quigg \& Rosner (67) & 81 & inverse & scatt. & - & $\overline{c \bar{c}}$ & 14.0 & +7 & 23 & 16 & \\
\hline 22 & Lichtenberg et al (49) & 88 & \multicolumn{2}{|c|}{$R^{0.1}$} & 3 & $\mathrm{~b} \overline{\underline{b}}$ & 16.0 & +32 & & & \\
\hline 23 & Crater et al (68) & 84 & C1 & $R$ & 1 & $\mathrm{~b} \overline{\mathrm{b}}$ & 16.3 & +27 & & & 3.8 \\
\hline 24 & Grotch et al (69) & 84 & $\mathrm{C} 2$ & $\boldsymbol{R}$ & 2 & $\mathrm{~b} \overline{\mathrm{b}}$ & 17.7 & & & 25 & 11.5 \\
\hline 25 & Martin (70) & 80 & \multicolumn{2}{|c|}{$R^{\beta}$} & 3 & $\mathrm{~b} \overline{\mathrm{b}}, \mathrm{c} \overline{\mathrm{c}}$ & 18.5 & +30 & 13 & & \\
\hline 26 & Grant et al (71) & 92 & \multicolumn{2}{|c|}{$R^{\beta}$} & 3 & $\mathrm{~b} \overline{\mathrm{b}}, \mathrm{cc} \overline{\mathrm{c}}$ & 21.1 & -11 & 16 & 283 & \\
\hline 27 & Heikkilä et al (72) & 83 & $\mathrm{CO}$ & $R^{2 / 3}$ & 3 & $c \bar{c}, b \bar{b}$ & 27.5 & -41 & 12 & & \\
\hline
\end{tabular}

As can be seen from the table, the potential models achieve an excellent precision of about $2 \mathrm{MeV}$ per mass level, that is about 0:02\% of the mass or $0.2 \%$ of the excitation energy. This is one of the most convincing experimental proofs of the quark structure of hadrons. The potential models are also able to describe $\Gamma_{\mathrm{ee}}$ ratios and radiative transition rates $\Gamma_{\mathrm{E} 1}$, with precision comparable to the experimental errors ( $6 \%$ and $12 \%$, correspondingly).

Some of the very first potentials applied to the b $\bar{b}$ system $(55,58$, 62, 66) (e.g. Models No. 9, 11, 16, 20) were tuned to the cic data but described the $b \bar{b}$ masses, including at that time unknown P-state masses, at a level of precision comparable to the most recent fits to the $Y$ data. This gives strong support for the QCD postulate of the flavor independence of the strong interactions. This independence was also demonstrated in a model independent way by Quigg \& Rosner (67), who used the inverse scattering method (No. 21). 
Most of the potentials attempt to test the other fundament of QCDasymptotic freedom of quarks. As the strong coupling constant of QCD becomes smaller for small interquark distances, the self-coupling of gluons becomes unimportant and the potential reduces to the familiar QED-like Coulomb potential, $V(R) \sim-\alpha_{\mathrm{s}} / R$ (denoted C0 in the Table $5)$, where $\alpha_{\text {'s }}$ is the strong coupling constant. In the Coulomb potential, the $2 \mathrm{~S}$ and the $1 \mathrm{P}$ states are degenerate, which is clearly not supported by the $b \bar{b}$ data. This implies that long-range nonperturbative effects in quark interactions are also important for the $\gamma$ system. The shape of the potential in the nonpcrturbative region has been calculated from QCD using numerical methods on the lattice (73). Its asymptotic behavior for large $R$ is linear; indeed, quark confinement demands that any potential form must contain a term that grows with $R$. The simplest QCD-motivated model (No. 17, 20) is just a sum of these two asymptotic behaviors and was proposed by Eichten et al (66) ("Cornell potential"). An updated fit of this model (49) (No. 14) does not yield such precise mass predictions as models that interpolate between the expected asymptotic QCD behavior. Bhanot \& Rudaz (58) (No. 11) proposed one of the early, and successful, models of this type.

The Coulomb singularity at the origin is softened by a logarithmic term, $V \sim-1 /(R \log R)$, if the running of the strong coupling constant is taken into account ( $\mathrm{Cl}$ in the table). Perhaps the simplest and the most successful potential proposed by Richardson (55) (No. 9) was based on this idea. By modifying the lowest order QCD formula for $\alpha_{\mathrm{s}}$, he obtained the desired smooth interpolation from the short-range perturbative potential to the long-range linear behavior. With just one free parameter tuned to the c $\bar{c}$ data, he managed to produce one of the most successful predictors for the $b \bar{b}$ system. Retuned to the more recent $b \bar{b}$ data by Fulcher (48), Richardson's potential gives the best fit to the clata (No. 1 and No. 5 in the table). Similar in shape is the potential proposed by Lichtenberg et al (49) ("Indiana" potential), also giving a good fit (No. 2).

The next-to-leading order QCD calculation (C2) must be performed to relate correctly the $\alpha_{\mathrm{s}}$ dependence on distance $R$ to the true QCD scale parameter (e.g. $\Lambda \overline{\mathrm{MS}}$ ), as first done by Buchmüller, Grunberg \& Tye (53) (No. 7). Models of Richardson and Buchmüller et al were also studied by other authors (No. 8, 24) $(54,69)$. Gupta, Radford \& Repko (56) went further, calculating even higher QCD corrections to the perturbative potential (C3). This model also describes the data very well (No. 4, 10) $(50,56)$.

Although the success of the QCD-motivated models is apparent, the spin-averaged masses can also be successfully described by purely phe- 
nomenological potentials. This approach was taken by Martin (70) (No. 25), who successfully described the level spacings in charmonium and vector states of the $Y$ system using a simple power law potential $V \sim$ $R^{\beta}(\beta \approx 0.1)$. In the limit of $\beta \rightarrow 0$ the power law potential becomes logarithmic; in this limit the mass spacing does not depend on quark mass. The logarithmic potential was studied by Quigg \& Rosner (62) (No. 16), who noticed that $M\left(Y^{\prime}\right)-M(Y) \approx 563 \mathrm{MeV} \approx M(\psi)-$ $M(\psi) \approx 590 \mathrm{MeV}$. In fact, the compact size of the $b \bar{b}$ states are such that $b$ quarks probe mostly the intermediate range potential, where the potential is approximately logarithmic and where the QCD-motivated models and purely phenomenological potentials coincide (see Figure 6).

The $\Gamma_{\text {ee }}$ widths of the $Y(n S)$ states are proportional to the wave function at the origin, and therefore are more sensitive to the short-distance potential. If the quark mass is tuned in the logarithmic or power law potentials to reproduce the measured $\Gamma_{\mathrm{ee}}$ 's, the radiative transition

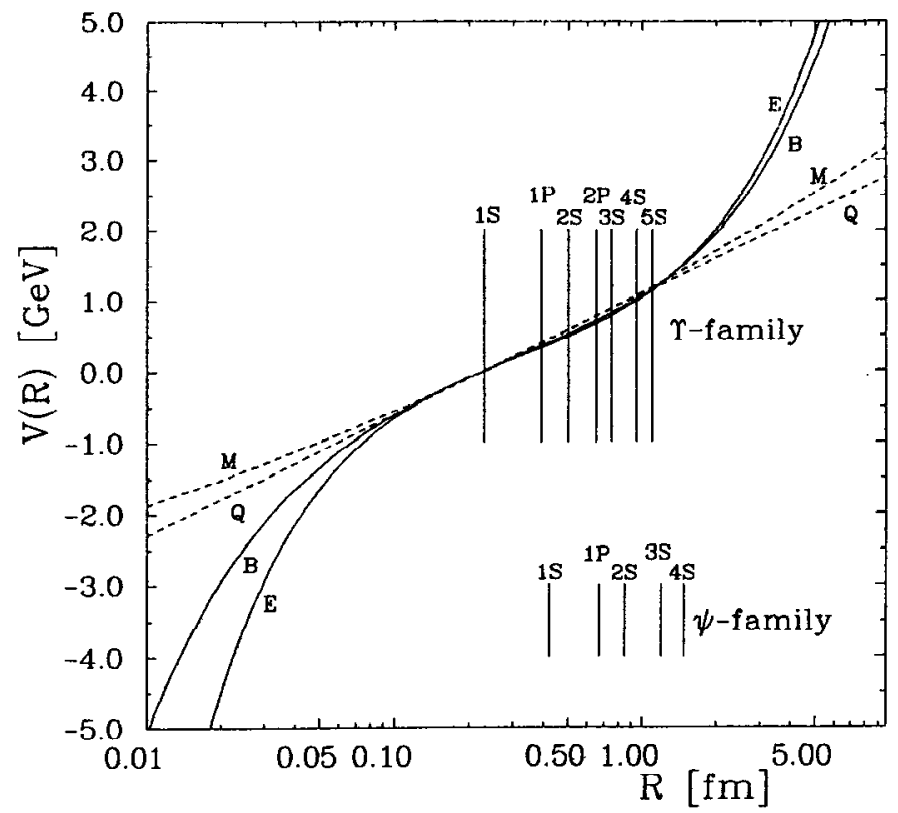

Figure 6 Shapes of various potentials as a function of interquark distance $R$, on a logarithmic scale, compared to sizes $\left(\bar{R}^{2} 1 / 2\right)$ of the $b \bar{b}$ and c $\bar{c}$ quarkonia, as calculated in one of the potential models. Solid lines show the QCD motivated models: $(E)$ Eichten et al (66), (B) Buchmüller et al. (53) Purely phenomenological potentials by Martin $(M)$ (70) and Quigg and Rosner $(Q)(62)$ are represented by dashed lines. All potentials have been shifted to cross zero at the $Y(1 S)$ radius. 
rates cannot be reproduced with the same set of parameters (71) (see No. 26). Also the mass fits for the logarithmic and Martin's potentials (No. 16, 22, 25, 26) $(62,49,70,71)$ are not as good as for the QCDmotivated models. Phenomenological models (49) that fit the masses, as well as the QCD-motivated models, all assume that the potential is singular at the origin (No. 3, 12).

Spin-independent relativistic corrections to the nonrelativistic mass calculations are expected to be of the order of $\overline{v / c}{ }^{2} \approx 8 \%$ in the $b \bar{b}$ system. Some authors $(54,65,69)$ attempted to implement perturbatively the first-order spin-independent corrections to the mass predictions in a consistent way (No. 8, 19, 24). Other authors $(59,60,68)$ tried to apply relativistic schemes different from the perturbed-Schrödinger calculations (No. 13, 15, 23). They do not improve the agreement with the data for the heavy quarkonia, but thanks to the nonperturbative relativistic calculations, these potential models have been successfully applied to mesons built up from the light quarks: $u, d$, and s. In addition, quantum effects of coupling bound $b \bar{b}$ states below the $B \bar{B}$ threshold to virtual $\mathbf{B}^{(*)} \mathbf{B}^{(*)}$ pairs can shift the masses of the quarkonium states (66). To demonstrate the effect of direct $Y(4 S)$ decays into $\mathrm{B} \overline{\mathrm{B}}$ pairs on the potential model predictions, we have included in Table 5 the following value: $\Delta M(4 \mathrm{~S})=\left[M(4 \mathrm{~S})_{\mathrm{th}}-M(4 \mathrm{~S})_{\mathrm{m}}\right]-\overline{\Delta M}$. Models that precisely predict masses below $\mathrm{B} \overline{\mathrm{B}}$ threshold consistently overestimate the $Y(4 S)$ mass by $30-50 \mathrm{MeV}$. Calculations of Heikkilä, Tórnqvist \& Ono (72) predicted mass shifts of $30-60 \mathrm{MeV}$ for the $b \bar{b}$ states below the $\bar{B} \bar{B}$ thresholds. However, these calculations did not lead to better mass fits (No. 27). Despite the simplicity of the totally nonrelativistic models, it may well be the case that the dominant spinindependent relativistic corrections and coupled channel effects can be absorbed into a redefinition of the potential parameters and quark masses, which may explain the success of these models.

\subsection{Electric Dipole Matrix Elements}

The radiative transition width from an initial state with quantum numbers $\mathrm{n} ; \mathrm{L} ; \mathrm{J}$; to the final state $n_{\mathrm{f}} L_{\mathrm{f}} J_{\mathrm{f}}$ can be calculated in a potential model in the long wavelength limit from

$$
\Gamma_{\mathrm{E} 1}=\frac{4}{3} e_{\mathrm{b}}^{2} \alpha C_{\mathrm{if}}\left(2 J_{\mathrm{f}}+1\right) E_{\gamma}^{3}\left|\left\langle n_{\mathrm{f}} L_{\mathrm{f}}|R| n_{\mathrm{i}} L_{\mathrm{i}}\right\rangle\right|^{2},
$$

where $\langle n \mathrm{~L}|$ denotes the radial wave function of the bound state, and $C_{\mathrm{f}_{\mathrm{i}}}$ is a factor that depends on the intial- and final-state quantum numbers. For transitions between $S$ and $P$ states, $C_{\mathrm{fi}}=1 / 9$. Note that in 
the nonrelativistic limit the wave functions depend only on the radial and orbital momentum quantum numbers, and therefore, the transition rates for different spins of the $P$ states differ only by the phase space factor: $\left(2 J_{f}+1\right) E_{\gamma}^{3}$.

The pattern of transition widths among the $b \bar{b}$ states can be understood from simple model-independent considerations. As the radial quantum number, $n$, determines the number of nodes of the wave function, the E1 matrix element $\left\langle n_{\mathrm{f}} L_{\mathrm{f}}|R| n_{\mathrm{i}} L_{\mathrm{i}}\right\rangle$ decreases with increasing $n_{\mathrm{i}}-n_{\mathrm{f}}$ because of cancellations between wave functions with different numbers of nodes. Small differences in the wave functions, due to different potential shapes or to relativistic corrections, may have a large impact on the cancellation mechanism. The phase space factor, $E_{\gamma}^{3}$, acts in the opposite direction, since the difference in the bound state masses increases with increasing $n_{i}-n_{\mathrm{f}}$.

For the $n \mathrm{~S}$ states, the experimentally measured radiative branching ratios, $B_{\gamma}$, can easily be converted to radiative widths, $\Gamma_{\gamma}=B_{\gamma} \Gamma_{\text {tot }}(n S)$ since the total widths of those states are independently determined. Total widths of the P states have not yet been measured independently of the radiative branching ratios (see the next section). However, we can still test the predicted ratio of the E1 matrix elements $\langle 1 \mathrm{~S}|R| 2 \mathrm{P}\rangle /$ $\langle 2 S|R| 2 \mathrm{P}\rangle$ since the total widths of the $\mathrm{P}$ states drop out in the ratio of the corresponding branching fractions. From the experimental $\Gamma_{\gamma}$ 's we have extracted values of the E1 matrix elements. In the nonrelativistic limit these elements are spin independent, so we averaged over the different spins of the $P$ states using experimental errors as weights. Table 6 compares the data ${ }^{4}$ with the theoretical predictions for the four observables: $\langle 2 \mathrm{P}|R| 3 \mathrm{~S}\rangle,\langle 1 \mathrm{P}|R| 2 \mathrm{~S}\rangle,\langle 1 \mathrm{P}|R| 3 \mathrm{~S}\rangle$, and $\langle 1 \mathrm{~S}|\overline{\mathrm{R}}| 2 \mathrm{P}\rangle /$ $\langle 2 S|\bar{R}| 2 \mathrm{P})$. The measurement of $B(3 S \rightarrow \gamma 1 \mathrm{P}) B(1 \mathrm{P} \rightarrow \gamma 1 \mathrm{~S})$ contains an experimentally unseparated mixture of different spins of the 1P states. Nevertheless, we can still extract $\langle\mathrm{IP}|R| 3 \mathrm{~S}\rangle$ by calculating: $[B(3 S \rightarrow \gamma 1 \mathrm{P}) B(1 \mathrm{P} \rightarrow \gamma 1 \mathrm{~S})] /\left[\sum_{J=0}^{J=2}(2 J+1) E_{\gamma}^{3}\left(3 S_{1} \rightarrow\right.\right.$ $\left.\left.1 \mathrm{P}_{J}\right) B\left(1 \mathrm{P}_{J \rightarrow \gamma} 1 \mathrm{~S}_{1}\right)\right]$, where $E_{\gamma}\left(3 \mathrm{~S}_{1} \rightarrow 1 \mathrm{P}_{J}\right)$ can be calculated from the measured masses of $3 S_{1}$ and $1 P_{J}$ states and $B\left(1 P_{J \rightarrow \gamma} 1 S_{1}\right)$ are experimentally measured. ${ }^{5}$ Comparison with the nonrelativistic calculations is straightforward. For relativistic calculations, in which the E1 matrix elements depend on the spins of the P states, we have calculated the spin-averaged value, using exactly the same procedure as applied to the data (i.e. again using weights corresponding to the experimental

\footnotetext{
${ }^{4}$ We have used only $J=2$ and $J=1$ to determine the ratio $\langle 1 S|R| 2 \mathrm{P}\rangle /\langle 2 \mathrm{~S}|R| 2 \mathrm{P}\rangle$ since the radiative cascade transitions via $J=0$ are not well established experimentally.

${ }^{5}$ For $J=0$ we assumed a value that is half of the experimental upper limit, with the error covering the range from zero to the upper limit value.
} 
Table 6 Comparison of $\mathrm{E} 1$ matrix elements (in $\mathrm{GeV}^{-1}$ ) predicted by the given potential model with measurements from $b \bar{b}$ data $^{a}$

\begin{tabular}{|c|c|c|c|c|c|c|c|c|}
\hline \multirow{4}{*}{$\begin{array}{l}\text { Data } \\
\text { Model }\end{array}$} & \multirow{3}{*}{\multicolumn{2}{|c|}{$\begin{array}{c}\langle 2 \mathrm{P}|R| 3 S\rangle \\
2.7 \pm 0.2\end{array}$}} & \multirow{3}{*}{\multicolumn{2}{|c|}{$\begin{array}{c}\langle 1 \mathrm{P}|R| 2 \mathrm{~S}\rangle \\
1.9 \pm 0.2\end{array}$}} & \multirow{3}{*}{\multicolumn{2}{|c|}{$\begin{array}{c}\langle 1 \mathrm{P}|R| 3 \mathrm{~S}\rangle \\
0.041 \pm 0.008\end{array}$}} & \multirow{3}{*}{\multicolumn{2}{|c|}{$\begin{array}{l}\langle 1 \mathrm{~S}|R| 2 \mathrm{P}\rangle \\
\langle 2 \mathrm{~S}|R| 2 \mathrm{P}\rangle \\
0.10 \pm 0.01\end{array}$}} \\
\hline & & & & & & & & \\
\hline & & & & & & & & \\
\hline & NR & rel & NR & rel & NR & rel & NR & rel \\
\hline Kwong \& Rosner (75) & 2.7 & & 1.6 & & 0.023 & & 0.13 & \\
\hline Fulcher (48) & 2.6 & & 1.6 & & 0.023 & & 0.13 & \\
\hline Büchmuller et al (53) & 2.7 & & 1.6 & & 0.010 & & 0.12 & \\
\hline Moxhay \& Rosner (54) & 2.7 & 2.7 & 1.6 & 1.6 & 0.024 & 0.044 & 0.13 & 0.15 \\
\hline Gupta et al (57) & 2.6 & & 1.6 & & 0.040 & & 0.11 & \\
\hline Gupta et al (56) & 2.6 & & 1.6 & & 0.010 & & 0.12 & \\
\hline Fulcher (76) & 2.6 & & 1.6 & & 0.018 & & 0.11 & \\
\hline Daghighian et al (61) & 2.8 & 2.5 & 1.7 & 1.3 & 0.024 & 0.037 & 0.13 & 0.10 \\
\hline McClary \& Byers (65) & 2.6 & 2.5 & 1.7 & 1.6 & & & 0.15 & 0.13 \\
\hline Eichten et al (56) & 2.6 & & 1.7 & & 0.110 & & 0.15 & \\
\hline Grotch et al (69) & 2.7 & 2.5 & 1.7 & 1.5 & 0.011 & 0.061 & 0.13 & 0.19 \\
\hline Grant et al (71) & 5.8 & & 3.3 & & 0.071 & & & \\
\hline
\end{tabular}

a "NR" denotes nonrelativistic calculations and "rel" refers to models with relativistic corrections.

errors). The models are ordered according to the quality of the mass fit, described in the previous section. The first model in Table 6 (75) uses the inverse scattering potential reconstructed from the measured values of $M_{\mathrm{cog}}$ and $\Gamma_{\mathrm{ce}}$.

There is very little model dependence (R.M.S. $=3 \%$ ) in the predictions for the E1 matrix elements for $3 \mathrm{~S} \rightarrow \gamma 2 \mathrm{P}$ and $2 \mathrm{~S} \rightarrow \gamma 1 \mathrm{P}$. A larger spread (R.M.S. $=10 \%$ ) can be found for the ratio of $2 \mathrm{P} \rightarrow \gamma 1 \mathrm{~S}$ and $2 \mathrm{P} \rightarrow \gamma_{2} \mathrm{~S}$. The predictions compare well with the data, and the relativistic corrections are generally small. As expected from the cancellation mechanism, the predictions for $3 \mathrm{~S} \rightarrow \gamma 1 \mathrm{P}$ are the most model dependent and the most sensitive to relativistic corrections. Excluding the anomalously high prediction by Eichten et al (66), the R.M.S. spread among different nonrelativistic models is still 50\%. All relativisitic calculations predict a large enhancement factor for this transition, bringing the theoretical predictions closer to the measured value. However, the correction is again strongly model dependent.

Relativistic corrections can also be tested by considering the spin dependence of the above matrix elements (excluding $3 \mathrm{~S} \rightarrow \gamma 1 \mathrm{P}$, for which the spin dependence was not measured). The spin dependence of the matrix elements is interesting because, like the fine splitting of levels discussed below, it probes the nature of the spin forces in strong interactions. Ratios of branching ratios are usually much better deter- 


\section{$356 \quad$ BESSON \& SKWARNICKI}

Table 7 Comparison of ratio of $E 1$ matrix elements squared between the $b \bar{b}$ data and relativisti potential models for different spins, $J$, of the $P$ states (in the nonrelativistic limit these ratios shoul be 1)

\begin{tabular}{|c|c|c|c|c|c|c|}
\hline \multirow[t]{3}{*}{ Model } & \multicolumn{2}{|c|}{$|\langle 2 \mathrm{P}|R| 3 \mathrm{~S}\rangle|^{2}$} & \multicolumn{2}{|c|}{$|\langle 1 \mathrm{P}|R| 2 \mathrm{~S}\rangle|^{2}$} & \multicolumn{2}{|c|}{$\frac{|(1 \mathrm{~S}|R| 2 \mathrm{P})|^{2}}{|\langle 2 \mathrm{~S}|R| 2 \mathrm{P}\rangle|^{2}}$} \\
\hline & $\underline{J=2}$ & $J=0$ & $J=2$ & $J=0$ & $J=2$ & $J=0$ \\
\hline & $J=1$ & $J=1$ & $J=1$ & $J=1$ & $J=1$ & $J=1$ \\
\hline Data & $1.17 \pm 0.04$ & $0.74 \pm 0.06$ & $0.92 \pm 0.11$ & $0.95 \pm 0.16$ & $1.33 \pm 0.26$ & $0.37 \pm 0.3$ \\
\hline Moxhay \& Rosner (54) & 1.05 & 0.90 & 1.05 & 0.89 & 1.14 & 0.68 \\
\hline Daghighian et al (61) & 1.15 & 0.86 & 1.16 & 0.85 & 1.74 & 0.47 \\
\hline McClary \& Byers (65) & 1.17 & 0.73 & 1.13 & 0.76 & 1.52 & 0.26 \\
\hline Grotch et al (69) & 1.13 & 0.84 & 1.13 & 0.85 & 1.42 & 0.59 \\
\hline
\end{tabular}

mined experimentally than absolute rates, and thus allow more precise tests. The comparison between data ${ }^{6}$ and relativistic calculations is shown in Table 7 . The data for $3 \mathrm{~S} \rightarrow \gamma 2 \mathrm{P}$ agree well with the prediction of all relativistic calculations that the $J=2$ rate should be enhanced and the $J=0$ rate should be suppressed relative to the $J=1$ rate. The experimental errors on $2 \mathrm{~S} \rightarrow \gamma \mathrm{IP}$ transitions are at present too large to test the relativistic corrections. Although the errors on the ratio of the ratios for $2 \mathrm{P} \rightarrow \gamma 1 \mathrm{~S}$ and $2 \mathrm{P} \rightarrow \gamma 2 \mathrm{~S}$ are also large, the data confirm a large relativistic suppression expected for the $3 \mathrm{~S} \rightarrow \gamma 1 \mathrm{P}_{0}$ (74).

\subsection{Hadronic Widths of the P States}

Since the potential models describe radiative widths for the main transitions in the $b \bar{b}$ system rather well, we can use these predictions to turn the experimentally measured $B\left(n_{\mathrm{P}} \mathrm{P}_{J} \rightarrow \gamma n_{\mathrm{S}} \mathrm{S}_{1}\right)$ into total or hadronic widths of the $1 \mathrm{P}_{J}$ and $2 \mathrm{P}_{J}$ states, ${ }^{7} \Gamma_{\text {tot }}=\Gamma_{\mathrm{EI}} / B^{\gamma}$ and $\Gamma_{\text {had }}=\Gamma_{\text {tot }}$ $-\Gamma_{\mathrm{E} 1}$. There is good agreement among the nonrelativistic models for matrix elements for these transitions. Averaging over eleven different calculations we find $\langle 1 \mathrm{~S}|R| 1 \mathrm{P}\rangle=1.2 \pm 0.2 \mathrm{GeV}^{-1},\langle 2 \mathrm{~S}|R| 2 \mathrm{P}\rangle=3.6$

${ }^{6}$ For the $3 \mathrm{~S} \rightarrow \gamma 2 \mathrm{P}$ transitions we have used only the CLEO-II data (42), as the CUSBII experiment (44) did not resolve the $J=2$ and $J=1$ lines; it was therefore impossible to estimate an error (which must be strongly affected by the correlation coefficient from the fitted line amplitudes, not reflected by the diagonal errors) on the ratio of branching ratios.

${ }^{7}$ The dipion widths for the $\chi_{\mathbf{b}}^{\prime} \rightarrow \chi_{\mathrm{b}}$ are usually ignored in these extractions. Including this effect is typically a $<5 \%$ correction to the direct hadronic width derived in this fashion for the $J=1$ state, and $<1 \%$ for the $J=0$ and $J=2$ states. 
$\pm 0.4 \mathrm{GeV}^{-1}$, and $\langle 1 \mathrm{~S}|R| 2 \mathrm{P}\rangle=0.06 \pm 0.007 \mathrm{GeV}^{-1}$. Unfortunately, relativistic calculations $(54,61,65,69)$ disagree by large factors not only on the magnitude of the corrections, but also on their signs. Using the nonrelativistic matrix elements averaged over different models, we obtain for the 1P state: $\Gamma_{\text {tot }}\left(\Gamma_{\text {had }}\right)=158 \pm 54(120), 93 \pm 29(61)$ and $>540$ (514) (90\% C.L.) keV for $J=2,1$, and 0 respectively, and for 2P: $\Gamma_{\text {tot }}\left(\Gamma_{\text {had }}\right)=55 \pm 10(26), 36 \pm 6(11)$ and $96_{-31}^{+90}(76) \mathrm{keV}$ for $J$ $=2,1,0$. The errors correspond to the uncertainty in the data only.

The hadronic decays of the $J=2$ and $J=0$ states are expected to be dominated by two-gluon emission; for the $J=1$ state, which is prohibited from decaying into two massless vector particles, q⿳亠口冋g decays dominate (17). It is expected that the gluon produced in the decay of the $J=1$ state will preferentially be emitted with very low energy. ${ }^{8}$ Such $q \bar{q} g$ decays are therefore expected to be topologically very similar to $q \bar{q}$ deca.ys from the continuum at the same center-of-mass energy.

The $J=0$ and $J=2$ decays are therefore order $\alpha_{\mathrm{s}}^{2}$ processes, the $J=1$ decay is order $\alpha_{\mathrm{s}}^{3}$. Unlike the S-state decays, however, which have a decay width that varies as $|\Psi(0)|^{2} / m_{\mathrm{b}}^{2}$, the P states, because of the angular-momentum barrier, have a lowest order rate that varies as the square of the first derivative of the radial wave function at the origin: $\left|\Psi^{\prime}(0)\right|^{2} / m_{\mathrm{b}}^{4}$. In comparing with perturbative QCD predictions, it is convenient to take a ratio of the hadronic widths for different $J$, for which $\left|\Psi^{\prime}(0)\right|^{2} / m_{\mathrm{b}}^{4}$ drops out. General arguments give, for the ratio of decay widths of the $J=0$ and $J=2$ states into two massless vector particles, a value of $15 / 4=3.75$, arising largely from the fact that the $J=2$ states can assume $J_{\mathrm{z}}$ polarization values that have the wrong quantum numbers for such decays. QCD corrections $(17,77)$ to this leading order prediction are large, $\approx 1+10 \alpha_{s} / \pi$. Furthermore, these corrections contain a singularity in the binding energy, which also upsets the QCD prediction for the $J=1$ width in the leading order. Therefore, the QCD predictions might be on shaky ground here, although a phenomenological prescription for overcoming these difficulties was recently suggested (78). From the above derived hadronic widths of the P states we find $\Gamma_{\text {had }}^{J=0} / \Gamma_{\text {had }}^{J=2}>4.3$ (90\% C.L.) for 1P and $2.9 \pm 1.6$ for $2 \mathrm{P}$, qualitatively following the expectations. Also the $J=1$ states are the narrowest in each multiplet, as expected from the higher power of $\alpha_{\mathrm{s}}$ involved in their annihilation.

\footnotetext{
${ }^{8}$ This can be phenomenologically understood as a consequence of the running of $\alpha_{s}$ and the fact that this decay is described as the emission of two gluons, one of which is virtual (for which a longitudinal polarization is allowed) and decays to a q⿳亠口冖 pair. As the lower energy gluon becomes softer, the coupling at the gluon emission vertex becomes larger, and the rate is increased.
} 


\subsection{Fine Structure of the P States}

Relativistic effects generate spin dependence of the quarkonia levels. Spin-spin interactions are responsible for splitting the singlet states $S=0$ from the center of gravity of the triplet states $S=1$ (hyperfine structure). Lattice QCD calculations indicate that chromomagnetic spin-spin forces have only a short-range component (79); therefore, hyperfine splitting can be substantial only for the $S$ states. The singlet $1 \mathrm{~S}$ and $2 \mathrm{~S}$ states and the singlet $1 \mathrm{P}$ state are established in the cic system $(80,89)$. Their masses confirm this expectation. No singlet $b \bar{b}$ states have been discovered experimentally yet. Fine structure, which is generated by spin-orbit and tensor forces, has been measured in the $1 \mathrm{P}$ and $2 \mathrm{P}$ levels of the $\mathrm{b} \overline{\mathrm{b}}$ system. The masses of the $\mathrm{P}$ states can be expressed as: $M_{J=2}=M_{\mathrm{cog}}+a_{\mathrm{LS}}-0.4 a_{\mathrm{T}}, M_{J=1}=M_{\mathrm{cog}}-a_{\mathrm{LS}}+$ $2 a_{\mathrm{T}}, M_{j=0}=M_{\mathrm{cog}}-2 a_{\mathrm{LS}}-4 a_{\mathrm{T}}$, where the spin-orbit $a_{\mathrm{LS}}$ and tensor $a_{\mathrm{T}}$ coefficients are related to the static $\left(V_{0}\right)$ and the spin-dependent potentials $\left(V_{2}, V_{3}\right)(63,81): a_{\mathrm{LS}}=\left\langle n \mathrm{P}\left|1 / \mathrm{R} \frac{\mathrm{d}}{\mathrm{d} R}\left(2 V_{2}-0.5 V_{0}\right)\right| n \mathrm{P}\right) / m_{\mathrm{b}}^{2}$ and $a_{\mathrm{T}}=\left\langle n \mathrm{P}\left|V_{3}\right| n \mathrm{P}\right\rangle /\left(12 m_{\mathrm{b}}^{2}\right)$. Most of the phenomenological models relate $V_{2}$ and $V_{3}$ to parts of the static potential $V_{0}$. In other approaches, these potentials are modeled independently of $V_{0}$. In either case, the Lorentz transformation properties of the exchange operator using a relativistic description, which reduces to $V_{0}, V_{2}$, and $V_{3}$ in the semirelativistic approach, must be specified. For example, vector-type interactions contribute to all three of the above potentials, increasing $a_{\mathrm{LS}}$, whereas scalar exchange gives rise to $V_{0}$ only, decreasing $a_{\mathrm{LS}}$.

The parameters $a_{\mathrm{LS}}$ and $a_{\mathrm{T}}$ can be extracted from the data and compared to theoretical predictions. Experimentally, we can determine most precisely the ratio $r$ of mass splittings: $r=\left(M_{J=2}-M_{J=1}\right) /$ $\left(M_{j=1}-M_{J=0}\right)$, for which the systematic uncertainty in the absolute mass scale cancels out. Cancellation of the theoretical mass scale in the ratio is also advantageous because the predictions for $r$ are more sensitive to the underlying principles of the models and less sensitive to their technical details (such as fine tuning of the quark mass $m_{\mathrm{b}}$ ). A comparison of the measured and predicted fine structure parameters for the $1 \mathrm{P}$ and $2 \mathrm{P}$ b $\bar{b}$ states is shown in Table 8 .

QCD calculations on the lattice have shown that the tensor interactions and one part of the spin-orbit interaction are short range (79). They may be identified with one gluon-exchange interaction of a vector type. The other part of the spin-orbit interaction has a long-range component. Data on the splitting of the $b \bar{b}$ states show $a_{L S}>a_{T}$; thus the experimental data are sensitive to the long-range confining potential. Models assuming that the vector type of interaction extends to the 
Table 8 Comparison of fine structure parameters of the $b \bar{b} \mathbf{P}$ states as measured experimentally and predicted by different models

\begin{tabular}{|c|c|c|c|c|c|c|}
\hline \multirow[b]{2}{*}{ Model } & \multicolumn{3}{|c|}{$x_{\mathrm{b}}(1 \mathrm{P})$} & \multicolumn{3}{|c|}{$\chi_{\mathrm{b}}(2 \mathrm{P})$} \\
\hline & $r$ & $a_{\mathrm{LS}}(\mathrm{MeV})$ & $a_{\mathrm{T}}(\mathrm{MeV})$ & $r$ & $a_{\mathrm{LS}}(\mathrm{MeV})$ & $a_{T}(\mathrm{MeV})$ \\
\hline \multirow[t]{2}{*}{ Data } & $0.65 \pm 0.03$ & $14.2 \pm 0.8$ & $3.0 \pm 0.3$ & $0.58 \pm 0.01$ & $9.4 \pm 0.2$ & $2.3 \pm 0.1$ \\
\hline & \multicolumn{6}{|c|}{ Scalar confinement } \\
\hline Fulcher (51) & 0.64 & 12.2 & 2.6 & 0.59 & 9.1 & 2.2 \\
\hline Hirano $(82)$ & 0.63 & 16.1 & 3.6 & 0.65 & 11.6 & 2.5 \\
\hline Gupta ct al (57) & 0.64 & 10.8 & 2.4 & 0.67 & 9.3 & 1.9 \\
\hline Ito $(59)$ & 0.57 & 14.2 & 3.5 & 0.67 & 10.7 & 2.2 \\
\hline Fulcher (50) & 0.67 & 12.0 & 2.5 & 0.68 & 9.9 & 2.0 \\
\hline Gupta et al (56) & 0.68 & 11.3 & 2.3 & 0.70 & 9.2 & 1.8 \\
\hline Fulcher (48) & 0.68 & 12.6 & 2.6 & 0.73 & 10.3 & 1.9 \\
\hline McClary \& Byers (65) & 0.45 & $\mathbf{I 7 . 3}$ & 5.3 & 0.47 & 14.6 & 4.2 \\
\hline Carlson et al (83) & 0.34 & 9.9 & 3.7 & 0.65 & 11.4 & 2.4 \\
\hline Büchmuller (84) & 0.76 & 19.8 & 3.5 & 0.75 & 15.3 & 2.8 \\
\hline Grotch et al (69) & 0.76 & 14.0 & 2.5 & 0.76 & 10.2 & 1.8 \\
\hline \multirow[t]{2}{*}{ Bander et al (60) } & 0.77 & 17.1 & 3.0 & 0.82 & 11.2 & 1.8 \\
\hline & \multicolumn{6}{|c|}{ Vector confinement } \\
\hline Bander et al (60) & 0.88 & 17.6 & 2.6 & 0.90 & 10.8 & 1.5 \\
\hline \multirow[t]{2}{*}{ Grotch et al (69) } & 1.00 & 22.2 & 2.6 & 0.96 & 15.9 & 2.0 \\
\hline & \multicolumn{6}{|c|}{ Other types of confinement } \\
\hline Franzini (86) & 0.62 & 14.1 & 3.2 & 0.61 & 9.6 & 2.2 \\
\hline Gupta et al (52) & 0.65 & 13.8 & 2.9 & 0.65 & 10.9 & 2.3 \\
\hline Crater et al (68) & 0.74 & 16.7 & 3.1 & 0.72 & 11.7 & 2.2 \\
\hline Moxhay \& Rosner (54) & 0.42 & 8.9 & 2.8 & 0.42 & 6.5 & 2.1 \\
\hline Hiller (87) & 0.95 & 12.4 & 1.6 & 0.90 & 10.8 & 1.5 \\
\hline Eichten \& Feinberg (63) & 0.96 & 15.3 & 1.9 & 1.00 & 10.5 & 1.3 \\
\hline
\end{tabular}

confining regime overestimate $a_{\mathrm{LS}}$ and $r$ (see Table 8). For a pure Coulomb potential $r=0.8$, whereas for both $\mathrm{P}$ triplets, the measured $r$ is significantly lower. To cancel out the Coulombic contribution, most of the authors assume that long-range interactions are of the effective scalar type. Scalar long-range interactions correspond to confinement by chromo-electric fields alone (84).

Recently, the fine structure of $2 \mathrm{P}$ states was measured with high precision $(42,44)$, showing that $r(2 \mathrm{P})<r(1 \mathrm{P})$, contrary to most of the model predictions (see Table 8). In models with vector and scalar exchanges only, such ordering can be achieved only by increasing the vector component $(85,86)$, which leads to predictions for absolute values of $r$ in disagreement with the data. This situation can be fixed on phenomenological grounds by allowing other types of exchange in longrange interactions (86). The best description of the $b \bar{b}$ data was obtained 
by a model (51) developed even before the new data on the 2P states was published, which assumed linear scalar confinement and employed higher order QCD calculations of the short-range potential $(56,57)$ (the QCD potential is of pure vector type only in the lowest order), as advocated by Pantaleone, Tye \& Ng (88).

\section{HADRONIC TRANSITIONS BETWEEN BOTTOMONIA}

\subsection{Background}

The study of $\Delta I=0$ dipion transitions of heavy quarkonia historically connects with the study in the 1960 s of $\pi \pi \rightarrow \pi \pi$ scattering. This was part of the larger effort to undestand the isospin structure of dipion interactions at low energies, dominated by the $I=1(\rho)$ pole. The transitions $\eta^{\prime} \rightarrow \eta \pi \pi, \psi^{\prime} \rightarrow \psi \pi \pi$, and $Y(3 S), Y(2 S) \rightarrow Y(1 S) \pi \pi$ are all examples of $\Delta I=0$ dipion interactions. The first decay is well studied (89); the pions fit reasonably well to a phase space mass spectrum. Following the discovery of the $\psi$ in 1974, with the subsequent observation of the transition $\psi^{\prime} \rightarrow \psi \pi \pi$ and measurements of the dipion properties (90), theoretical attention turned to the challenge of providing an acceptable description of the observed data. For the typical transition energies considered in bottomonium transitions (0.3-0.9 $\mathrm{GeV}$ ), the dipion processes we consider here are sufficiently "soft" so as to be difficult to treat perturbatively.

Typically, the decay $\mathrm{q} \overline{\mathrm{q}} \rightarrow \mathrm{q} \overline{\mathrm{q}}^{\prime} X$, where $\mathrm{q} \overline{\mathrm{q}}$ is a heavy quarkonium state $\left[\psi^{\prime}, Y(2 S), Y(3 S)\right], \mathrm{q} \bar{q}^{\prime}$ is a daughter state [Y(1S), $\psi$, for example], and $X$ is some hadronic state (usually $\pi \pi$, or less frequently, $\eta$ ), is treated as the factorizable product of two independent processes: one describing the transition of $q \bar{q}$ to $q \bar{q}^{\prime}$ with an operator $H_{1}$, and the other describing the production of the state $X$ from the vacuum with an operator $H_{2}$. For the dipion transition from the $Y(3 S)$ to the $Y(1 S)$, for example, we assume

$$
\Gamma\left[Y(3 S) \rightarrow Y(1 S) \pi^{+} \pi^{-}\right] \propto\left|\left\langle 1 S\left|H_{1}\right| 3 S\right\rangle\left\langle\pi^{+} \pi^{-}\left|H_{2}\right| 0\right\rangle\right|^{2} .
$$

Although nonperturbative, the hadronic transitions between heavy quarkonia lend themselves to a treatment similar to the modeling of the electromagnetic radiative transitions, in the context of a "multipole" expansion scheme as first outlined by Gottfried (91). The radiative transitions already discussed are dominated by the electromagnetic E1 transition matrix element; for QCD we can write the transition between heavy quarkonia as a multipole expansion of the gluon color 
field in the expansion parameter $\mathbf{k} \cdot \mathbf{R}$, where $\mathbf{k}$ is the gluon momentum and $\mathbf{R}$ its spatial coordinate. Such an approach should be valid for $\mathbf{k} \cdot \mathbf{R}$ $\ll 1$. Taking a typical value of interquarkonium distance $\langle R\rangle$ to be $1 / 500 \mathrm{MeV}$, and the value of $k$ to be one half the transition energy, the expansion parameter ${ }^{9}\langle k \cdot R\rangle$ is of order 0.3 for $Y(3 S) \rightarrow Y(2 S) \pi^{+} \pi^{-}$. For $Y(3 S) \rightarrow Y(1 S) \pi \pi$, however, it can approach unity, which suggests a possible breakdown of the applicability of this model.$^{10}$ In this model, the interaction Hamiltonian $H_{1}$ for each gluon then takes the form ${ }^{11}$

$$
H_{\text {int }}=Q_{a} A_{a 0}(0, t)-\left(\mathbf{d}_{a} \cdot \mathbf{E}_{a}\right)(0, t)-\left(\mathbf{m}_{a} \cdot \mathbf{B}_{a}\right)(0, t),
$$

where $a=$ gluonic color subscript, $A=$ time component of gluonic four-potential, $Q=$ total color charge, $\mathbf{E}=$ chromoelectric field, $\mathbf{B}=$ chromomagnetic field, $\mathbf{d}=$ chromoelectric dipole moment, and $\mathbf{m}=$ chromomagnetic dipole moment.

Since the first term operates on the monopole color charge of the state in question, and since $Y$ 's are colorless, we have $Q_{a} A_{a 0}(0, t)|Y\rangle$ $=0$ trivially. The lowest order surviving terms are therefore electric dipole and the magnetic dipole term, respectively.

Unlike radiative electromagnetic transitions, single-gluon emission is prohibited by color considerations. The lowest order allowed transition is then the emission of two gluons. Since the gluons are vector particles, this gives selection rules for the dipion transitions. Whereas photon transitions connect states with different parities, lowest order two-gluon. transitions connect states with the same parity. $S \rightarrow S$ transitions from vector $Y$ to lower lying vector $Y$ states are therefore allowed, while $\mathrm{S} \rightarrow \mathrm{P}$ transitions from the $Y$ to the $\chi_{\mathrm{b}}$ states are disallowed. In the multipole model, since the chromomagnetic piece is masssuppressed, the lowest order allowed transition involves the emission of two E1 gluons.

For dipion transitions, Yan (92), collaborating with Kuang (96), and their work later extended by Zhou \& Kuang (97), estimated the magnitude of the second piece of the product matrix element, the hadronization term of the transition amplitude. This term can be written as proportional to $\left\langle\mathrm{X}\left|\alpha_{\mathrm{s}} \mathrm{E} 1_{1} \mathrm{E} 1_{2}\right| 0\right\rangle$. An immediate consequence of this approach is the suppression of the case $\mathrm{X}=\eta$ relative to $\mathrm{X}=\pi \pi$.

\footnotetext{
${ }^{9}$ We remind the reader that the contributions of the higher order terms go as $(\mathbf{k} \cdot \mathbf{R})^{\mathrm{n}}$.

${ }^{10}$ Certainly, for $\eta^{\prime} \rightarrow \eta \pi \pi$, the applicability of the multipole model is undermined by the large value of $\langle R\rangle$ expected for the typical spatial scale.

${ }^{11}$ The gluon field potential can be written as $\Phi(\mathbf{R})=Q_{\mathrm{a}} / R+$ bfk $\cdot \mathbf{R} / R^{3}+\ldots$, or as a Taylor's expansion: $\Phi(\mathbf{R})=\Phi(0)+\mathbf{R} \cdot \nabla \Phi(0)+\ldots$; the correspondence between the dipole terms is then clear when the latter equation is multiplied through by the color charge $Q_{\mathrm{a}}$.
} 
The former system has quantum numbers prohibited for two E1 gluons, and proceeds in lowest order as $\mathrm{E} 1 \cdot \mathrm{M} 2$, or $\mathrm{M} 1 \cdot \mathrm{M} 1$ in the multipole expansion. Since the mass dependence of the chromomagnetic transitions varies as $m^{-4}$ ( $m$ is the quark mass), the multipole model therefore predicts that the ratio for $B[Y(2 S) \rightarrow Y(1 S) \eta] / B[Y(2 S) \rightarrow Y(1 S) \pi \pi]$ should be substantially smaller than the ratio $B\left(\psi^{\prime} \rightarrow \psi \eta\right) / B\left(\psi^{\prime} \rightarrow \psi \pi \pi\right)$ $\approx 0.11$. By contrast, if the ratio of $\pi \pi$ to $\eta$ transitions were governed by phase space alone, the $\eta$ transition would be about $15 \%$ of the $\pi \pi$ transition for $Y(2 S) \rightarrow Y(1 S)$.

Rather than writing the gluonic degrees of freedom in terms of specific multipoles, Novikov, Shifman, Voloshin \& Zakharov (NSVZ) (93-95) used the general form of the QCD field tensor $G_{\mu \nu}^{\mathrm{a}}$, an extension of the familiar energy-stress tensor of QED. Setting $m^{\pi} \rightarrow 0$, they obtain a hadronization matrix element as $\left\langle\pi \pi\left|\alpha_{s} G_{\mu \gamma} \cdot G_{\nu \lambda}\right| 0\right\rangle$. In the NSVZ approach, as well as in the Yan approach, the hadronization matrix element can be constrained by current algebra, partial conservation of the axial current (PCAC), and gauge invariance. The essential mass dependence of the matrix element is very similar in both cases. The multipole approach, for instance, gives a matrix element (M.E.) depending on two parameters $(A, B)$, which varies as M.E. $\propto$ $A \mathbf{q}_{1}^{\mu} \mathbf{q}_{2_{\mu}}+B \mathrm{q}_{1_{0}} \mathrm{q}_{2_{0}}$, where $\mathrm{q}_{\mu}$ are the pion four-momenta, and the parameters $A$ and $B$ are independent of energy scale and should therefore be the same for $c \bar{c}$ and $b \bar{b}$. A value of $B=0$ obtains when the pions are emitted isotropically (i.e. S-wave). Both models give vanishing matrix elements (the "Adler" zero) for pion momenta approaching zero, and both favor large values of $m_{\pi \pi}$.

Yan et al determine the model parameters phenomenologically from a fit to $\psi^{\prime} \rightarrow \psi \pi^{+} \pi^{-}$. In NSVZ, the model parameters describing the gluon hadronization are obtained from "first-principles," combining perturbative QCD with a value for the pion form factor, which specifies the gluonic fraction of the pion.

Both approaches gave dipion mass distributions that could accommodate very well the data from $\psi^{\prime} \rightarrow \psi \pi \pi$, whereas a phase space distribution gave a poor fit to the data. Although the dipion mass shapes should be similar in extrapolating from the $\psi$ system to the $Y$ system, the predicted partial widths are generally smaller for $Y$ transitions than $\psi$ transitions. This is easily understood in the multipole model: Since the lowest order matrix element varies as: $\langle f|R| i\rangle$, and since the $b \bar{b}$

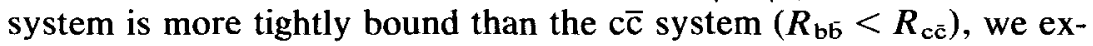
pect the partial widths for the hadronic decays to be smaller for bottomonium than for charmonium. 
The multipole model allows other straightforward predictions. For example, despite the fact that multipole rates generally increase with available space, varying as $k^{3}$, the partial width for $Y(3 S) \rightarrow Y(1 S) \pi \pi$ was predicted to be smaller than for $Y(2 S) \rightarrow Y(1 S) \pi \pi$, because of zeros in the $Y(3 S)$ wave function, and consequently small value of the $Y(1 S)-Y(3 S)$ overlap integral. This is the same suppression already noted for photon transitions between $3 \mathrm{~S}$ and $1 \mathrm{P}$ relative to $3 \mathrm{~S}$ and $2 \mathrm{P}$. Differences between the two models are sharpest in decays other than the lowest order $S \rightarrow S$ transitions, as shown in Table 9. We note that the original Yan model prediction for the dipion $Y(1 D)$ transitions is substantially higher than the value one would expect given the angular momentum barrier that must be crossed in these D-wave decays (92).

\subsection{Experimental Procedure}

The principal experimental observables are therefore $(a)$ the partial widths for the transitions between bottomonia, and $(b)$ the Dalitz plot variables: $\pi \pi$ - and $Y \pi$-invariant mass spectra and the angular distributions between final-state particles. To measure the transition $Y(3 S) \rightarrow Y(I S) \pi \pi$, for example, where the $Y(3 S)$ is produced at rest, one can use the constraint that the $Y(1 S)$ energy can be inferred directly from the ineasurement of the pion four-momenta to calculate the mass recoiling against the dipion system. As with the $\gamma \gamma$ cascades (see Section 4), one differentiates the "exclusive" case in which the $Y$ decays to a clean, background-free topology, such as $\mu^{+} \mu^{-}$or $\mathrm{e}^{+} \mathrm{e}^{-}$, from the "inclusive" case in which all events are accepted, and one calculates the mass recoiling against all oppositely signed dipion pairs.

\subsection{Branching Ratios and Partial Widths}

The mass spectra recoiling against dipions for $\pi^{+} \pi^{-}$and $\pi^{0} \pi^{0}$ respectively, from the CLEO-II experiment $(98,99)$, are shown in Figure 7.

Table 9 Comparison of Yan vs NSVZ predictions for various transitions between botomomia

\begin{tabular}{|c|c|c|}
\hline Decay process & Yan model & NSVZ \\
\hline$Y(1 \mathrm{D}) \rightarrow Y(\mathrm{IS}) \pi^{+} \pi^{-}$ & $24 \mathrm{keV}$ & $0.07 \mathrm{keV}$ \\
\hline$Y(3 S) \rightarrow \pi^{+} \pi^{-} h_{b}\left(1^{1} P_{1}\right)$ & $0.02 \mathrm{keV}$ & $<0.01 \mathrm{keV}$ \\
\hline$\frac{Y(3 S) \rightarrow \pi^{0} h_{b}\left(1^{1} P_{1}\right)}{Y(3 S) \rightarrow \pi^{+} \pi^{-} h_{b}\left(1^{1} P_{1}\right)}$ & $0.05-0.5$ & 20 \\
\hline
\end{tabular}



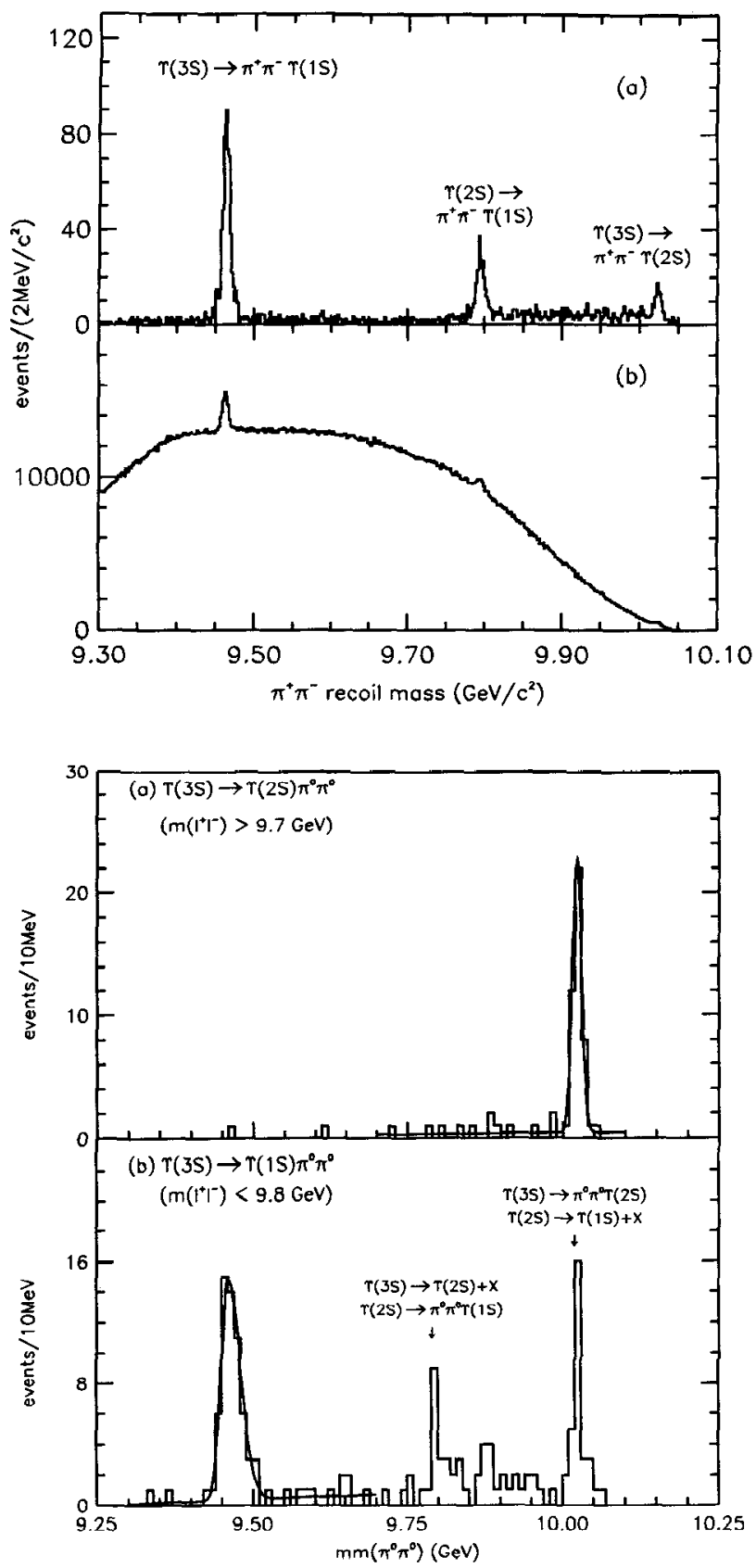

Figure 7 (Upper): $\pi^{+} \pi^{-}$recoil mass spectra from CLEO-II using data taken at the peak of the $Y(3 S)$ for $(a)$ exclusive transitions, and $(b)$ inclusive transitions. The transitions are as indicated. (Lower): $\pi^{0} \pi^{0}$ recoil mass spectrum for exclusives with the indicated cuts on dilepton mass $(98,99)$. 
Table 10 Branching ratios and rates of $\pi \pi$ transitions of bottomonia

\begin{tabular}{|c|c|c|c|c|}
\hline \multirow[b]{2}{*}{ Mode } & \multirow[b]{2}{*}{ Experiment } & \multirow{2}{*}{$\begin{array}{l}\text { Branching } \\
\text { ratio }(\%)\end{array}$} & \multicolumn{2}{|c|}{ Rates (keV) } \\
\hline & & & Result & Zhou-Kuang \\
\hline$Y(2 S) \rightarrow \pi^{0} \pi^{0} Y(1 S)$ & PDG 92[89] & $8.8 \pm 1.1$ & $3.8 \pm 0.8$ & \\
\hline$Y(2 S) \rightarrow \pi^{+} \pi^{-} Y(\mathrm{tS})$ & PDG 92 & $18.5 \pm 0.8$ & $7.6 \pm 1.6$ & \\
\hline$Y(3 S) \rightarrow \pi^{0} \pi^{0} Y(1 S)$ & $\begin{array}{l}\text { CLEO-II } \\
\text { CUSB-II } \\
\text { World Average }\end{array}$ & $\begin{aligned} 1.99 & \pm 0.34 \\
2.2 & \pm 0.5 \\
2.06 & \pm 0.28\end{aligned}$ & $0.50 \pm 0.07$ & 0.56 \\
\hline$Y(3 S) \rightarrow \pi^{+} \pi^{-} Y(\mathrm{IS})$ & $\begin{array}{l}\text { PDG } 92 \\
\text { CLEO-II } \\
\text { CUSB-II } \\
\text { New World Average }\end{array}$ & $\begin{array}{l}4.48 \pm 0.29 \\
4.52 \pm 0.35 \\
4.46 \pm 0.60 \\
4.49 \pm 0.21\end{array}$ & $1.10 \pm 0.05$ & 1.1 \\
\hline$Y(3 S) \rightarrow \pi^{0} \pi^{0} Y(2 S)$ & $\begin{array}{l}\text { CLEO-II }^{\mathrm{a}} \\
\text { CUSB-II } \\
\text { New World Average }\end{array}$ & $\begin{aligned} 2.16 & \pm 0.4 \\
1.7 & \pm 0.54 \\
2.00 & \pm 0.32\end{aligned}$ & $0.49 \pm 0.076$ & $0.13 \pm 0.07$ \\
\hline$Y(3 S) \rightarrow \pi^{+} \pi^{-} Y(2 S)$ & $\begin{array}{l}\text { PDG } 92 \\
\text { CLEO-II* } \\
\text { CUSB-II } \\
\text { New World Average }\end{array}$ & $\begin{aligned} 2.1 & \pm 0.4 \\
3.12 & \pm 0.5 \\
4.5 & \pm 0.84 \\
2.74 & \pm 0.3\end{aligned}$ & $0.66 \pm 0.08$ & $0.2 \pm 0.1$ \\
\hline$Y(3 S) \rightarrow Y(2 S)+X$ & $\begin{array}{l}\text { World Average } \\
\text { CLEO-II }\end{array}$ & $\begin{aligned} 10.9 & \pm 1.3 \\
10.23 & \pm 1.05\end{aligned}$ & $2.43 \pm 0.22 \pm 0.29$ & \\
\hline
\end{tabular}

a The CLEO collaboration are also able to derive estimates for the transition rates for $\gamma(3 S) \rightarrow \gamma(2 S)+x$ by performing a hand scan of the events it reconstructs in $Y(3 S) \rightarrow Y(2 S)+X, Y(2 S) \rightarrow Y(1 S) \pi^{+} \pi^{-}, Y(1 S) \rightarrow l^{+} l^{-}$, and using the unitarity constraint that the sum of the dipion transitions plus the radiative transitions must saturate the overall $Y(3 S) \rightarrow Y(2 S)+X$ decay rate to determine $X$. These values have been compiled along with the direct observations of the $Y(3 S) \rightarrow Y(2 S) \pi^{0} \pi^{0}$, or $Y(3 S) \rightarrow \pi^{+} \pi^{-}$transitions.

Clear signals are evident at the masses corresponding to the transitions indicated in the figure. ${ }^{12}$ Branching ratios are calculated based directly on the number of events found in each peak; a summary is given in Table 10 along with recent measurements by the CUSB-II (100) collaboration. In this table, the Zhou-Kuang results are obtained with the multipole expansion model, extended to take into account coupled channel effects. The branching ratios for the $Y(2 S) \rightarrow \pi \pi Y(1 S)$ were compiled by the Particle Data Group from measurements made by the ARGUS (101), CLEO-I (102), CUSB-I (103), and Crystal Ball (104) collaborations.

Isospin conservation requires the $\pi \pi$ system to be in an $I=0$ state in all of these transitions. The square of the matrix elements for the $\pi^{+} \pi^{-}$transitions should then be double those for the $\pi^{0} \pi^{0}$ transitions. The branching ratios also depend on the available phase space, which is somewhat larger for the $\pi^{0} \pi^{0}$ transitions. The ratios of available phase space $\left(\pi^{0} \pi^{0} / \pi^{+} \pi^{-}\right)$are 1.36 for $Y(3 S) \rightarrow \pi^{0} \pi^{0} Y(2 S)$ and 1.02 for

\footnotetext{
${ }^{12}$ Because of the poor signal-to-noise ratio the $\pi^{0} \pi^{0}$ transitions cannot be studied inclusively.
} 
$Y(3 S) \rightarrow \pi^{0} \pi^{0} Y(1 S)$. The measurements to date are generally consistent with this expectation, with the exception of $Y(3 S) \rightarrow \pi^{+} \pi^{-} Y(2 S)$, where the old World Average [dominated by the earlier CLEO-I measurement (105)] is conspicuously low compared to the newer measurements. Curiously, despite an inability to match the dipion mass distributions for the $Y(3 S) \rightarrow Y(1 S)$ transitions (see below), the multipole approach gives a better match for this partial width than for $Y(3 S)$ $\rightarrow \mathrm{Y}(2 \mathrm{~S})$.

\subsection{Angular Distributions}

In $\mathrm{e}^{+} \mathrm{e}^{-}$machines, the bottomonia resonances are produced polarized, with their spin axis lying along the beam axis. The hadronic decays of the $Y(3 S)$ must obey conservation of total angular momentum, as well as angular momentum along this axis. In addition to the polarization of the daughter $Y$, additional degrees of freedom are provided by the possibility of orbital angular momentum between the pions themselves, or orbital angular momentum between the dipion system and the daughter $Y$, subject to the usual selection rules of strong decays. Predictions for the populations of the allowed angular momentum states have been made for both the $\psi$ system as well as the $Y$ system $(106,107)$. All measurements to date, from ARGUS, CLEO, and CUSB give strong evidence that the daughter $Y(1 S)$ is indeed polarized along the beam axis in the dipion $Y$ transitions, and are consistent with an $\mathrm{S}$-wave decay. The other allowed amplitude is $\mathrm{D}$-wave ( $\mathrm{P}$-wave is excluded by the requirement that the dipions' wave function be symmetric, combined with the fact that the dipions must be in an $I=0$ state). Convincing evidence for a large $\mathrm{D}$-wave component of the dipion system has not yet been presented, although it has received some theoretical attention recently (108), and suggestions for anisotropy are found in the $Y(3 S) \rightarrow Y(1 S) \pi^{+} \pi^{-}$data (98). Mapping out the ratio of $D$ - to S-wave amplitudes as a function of dipion mass in the $Y(3 S)$ system is a project requiring substantially more statistics than have been accumulated to date; expectations are that a D-wave amplitude would be more observable at low values of invariant mass, corresponding to higher energy release in the $\mathrm{Y}(3 \mathrm{~S})$ decay.

\subsection{Dipion Mass Spectra}

The dipion mass spectra are calculated directly from the pion fourmomenta by CLEO, or from an energy-momentum event fit, by the CUSB collaboration. As stated before, the invariant mass spectra are expected to peak at high mass values. This is, in fact, what is observed for the transition $Y(2 S) \rightarrow Y(1 S) \pi^{+} \pi^{-}$, as shown in Figure 8, and is 


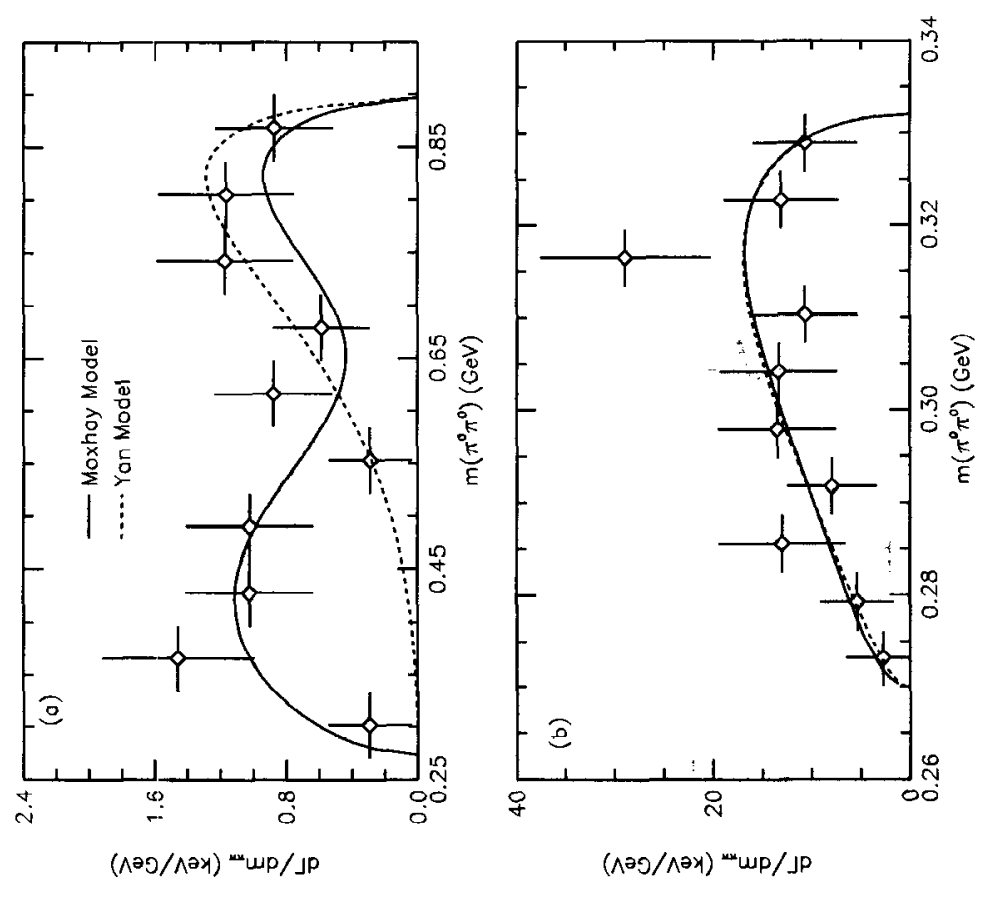

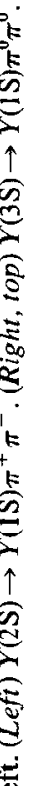

$(\Lambda ә J / \Lambda ә x)=m p / j \beta$

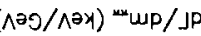

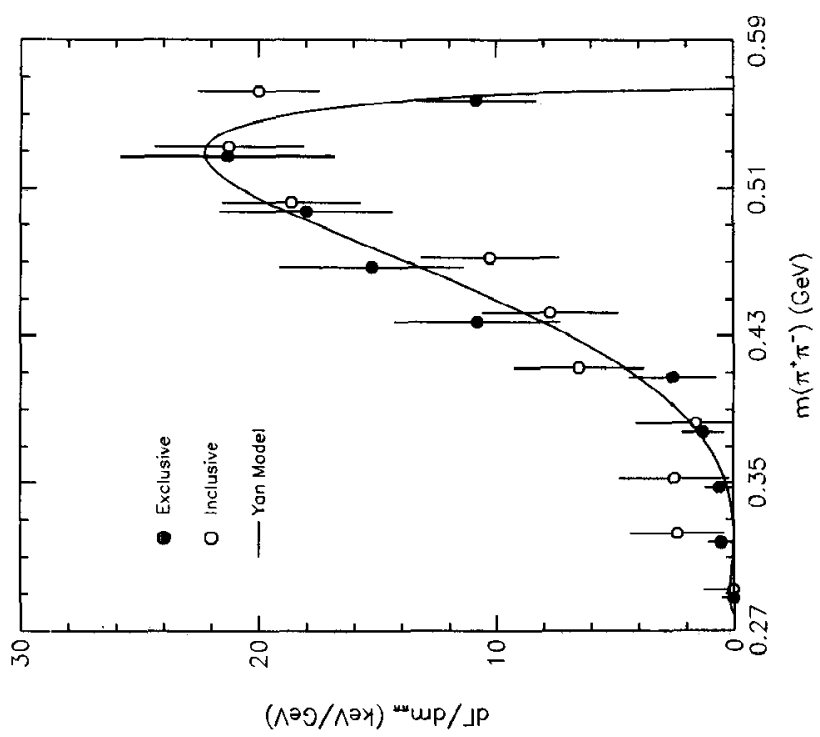

\%

$\ddot{\overrightarrow{\mathrm{B}}} \stackrel{0}{\circ}$

ह

:

되 둔

된

串

통 웡

은

宅

을

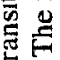

5

$>$ is

总

氙恣

总 $\uparrow$

के

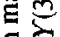

을

$\infty 8$

$\stackrel{5}{5}$

$(\Lambda \partial \supset / \wedge \partial x) \sim u p /\lrcorner P$ 
entirely consistent with an exhaustive study of this process by the ARGUS collaboration (101). Also shown in Figure 8 are the $\pi^{0} \pi^{0}$ mass spectra for $Y(3 S) \rightarrow Y(1 S) \pi^{0} \pi^{0}$ and $Y(3 S) \rightarrow Y(2 S) \pi^{0} \pi^{0}$.

The CUSB dipion mass spectra (100) are shown in Figure 9 for $Y(3 S)$ $\rightarrow Y(1 S) \pi^{-} \pi^{+}$and $Y(3 S) \rightarrow Y(2 S) \pi^{-} \pi^{+}$. The data from both the CLEO and CUSB experiments show peaking at high mass for the $Y(3 S)$ $\rightarrow Y(2 S) \pi \pi$ transitions, consistent with expectation for $S \rightarrow S$ transitions. This is the process for which the multipole expansion model, owing to the smallness of the expansion parameter, claims to have the greatest predictive power. However, the $\pi^{0} \pi^{0}$ and $\pi^{+} \pi^{-}$invariant mass distributions in the $Y(3 S) \rightarrow \pi \pi Y(1 S)$ transition show a double bump structure that disagrees with the gluon field multipole expansion model as well as with the expectation that the matrix element for a transition with these quantum numbers should approach zero at threshold. This is perhaps an indication that the average value of $Q^{2}$ is too large to make predictions reliably using the multipole model.

Several unsuccessful theoretical attempts have been made to remedy this discrepancy. Almost all are phenomenologically based and involve introducing another amplitude into the matrix element, for which there may or may not be any physical evidence. These approaches include the following: (a) Taking into account the known $\pi \pi$ final-state interaction (109); (b) Introducing $\pi \pi$ and/or $Y \pi$ resonances $(95,110)$, which would be evidenced by an (as yet unseen) enhancement in the inclusive pion momentum spectrum from the parent particle; and $(c)$ positing that the dipion mass spectrum is dominated by a scalar resonance $(\epsilon)$ (111); here predictions for branching ratios were derived that agreed with experiment, but the $Y(3 S) \rightarrow Y(1 S)$ dipion mass spectrum could not be matched (such resonances are not, however, observed in direct $\pi \pi$ scattering experiments). In addition to these approaches, a variety of coupled channel type approaches were put forward: $(a)$ Lipkin \& Tuan (112) suggested that the $\pi \pi$ invariant mass distribution in the $Y(3 S) \rightarrow \pi \pi Y(1 S)$ transition is due to coupling to intermediate BB* states; $(b)$ Moxhay (113) showed that a generic, constant complex amplitude-presumably coupling to virtual $\mathbf{B} \overline{\mathbf{B}}$ states and interfering with the multipole expansion amplitude-could reproduce the experimental $\pi \pi$ mass distribution available at that time, and (c) Zhou \& Kuang (97), working within the multipole framework, took into account coupled channel effects interfering with the multipole amplitude by writing explicitly the amplitude due to intermediate $\overline{B B}^{*}+\overline{\mathrm{B}} \mathrm{B}^{*}$ states. Both the Lipkin \& Tuan (112) and the Moxhay (113) models can be considered as special cases of the Zhou-Kuang approach. The free parameters in their calculation are the magnitude and phase of the multipole expansion term relative to the intermediate channels they consider. As in 

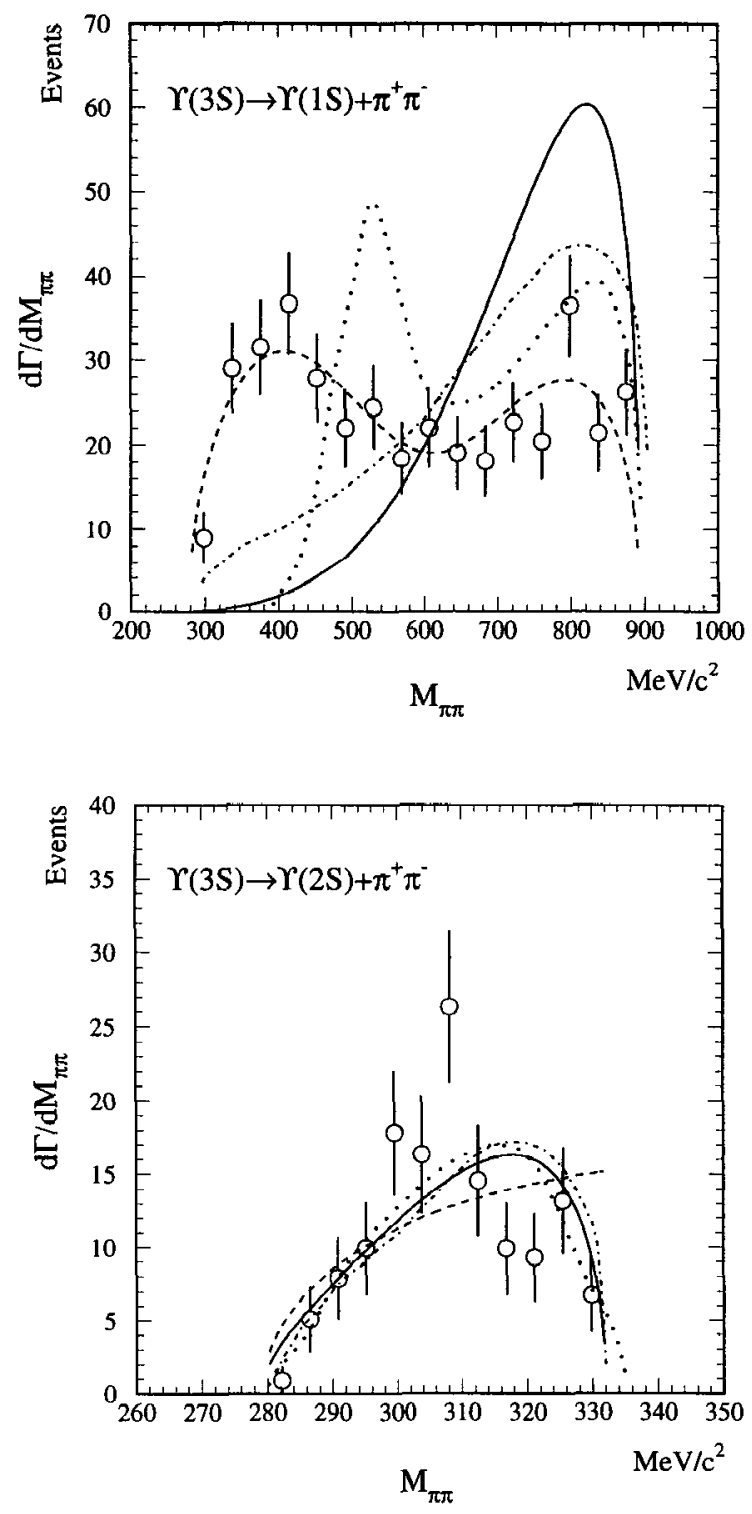

Figure 9 Dipion mass spectra from the CUSB experiment for the indicated transitions. The fits are as follows: Yan-Kuang spectrum (solid lines), Moxhay model (dashed lines), Zhou-Kuang model (dot-dashed line), and Bélanger et al (109) (dotted line) (97). 
the original Yan model, the parameters are again obtained from fitting $\mathrm{d} \Gamma / \mathrm{d} m_{\pi \pi}$ for the $\psi^{\prime} \rightarrow \psi \pi \pi$ transition (90). Their model predicts $\pi \pi$ decay rates larger than those calculated by Kuang \& Yan (96) and in closer agreement with the experimental ones. However, according to their model, the net effect of including these coupled channel effects turns out to be at least one order of magnitude smaller than the multipole expansion term and thus is inconsistent with the experimental $m_{\pi \pi}$ distribution in $Y(3 S) \rightarrow Y(1 S) \pi \pi$ decay.

\subsection{Unanswered Questions}

Aside from a first-principles explanation of the dipion mass spectrum in the $Y(3 S) \rightarrow Y(1 S) \pi \pi$ spectrum (such a three-body decay does not, unfortunately, easily lend itself to lattice gauge techniques), much experimental work remains. Among the dipion transitions one would like to observe are the following five. (a) $Y(4 S) \rightarrow Y(15)$ which is expected to be extremely suppressed owing to the small wave function overlap between the initial and final states. ( $b$ ) The $\eta$ transitions between the $S$ states, or one of the two dipion transitions involving the singlet $1{ }^{1} \mathrm{P}_{1}$ state: the isospin-violating decay $Y(3 S) \rightarrow \pi^{0} h_{b}\left(1^{1} P_{1}\right)$, or $Y(3 S) \rightarrow$ $\pi^{+} \pi^{-} h_{b} \cdot{ }^{13}$ We note that the isospin-violating decay $h_{c}\left(1^{1} P_{1}\right) \rightarrow \pi^{0} \psi$ was recently observed by the E760 collaboration (115), and the isospinviolating decay $\psi^{\prime} \rightarrow \pi^{0} \psi$ has also been observed. (c) The dipion transitions between the $\chi_{\mathrm{b}}$ states: $\chi_{\mathrm{b}}^{\prime} \rightarrow \chi_{\mathrm{b}} \pi \pi$. Owing to the larger total widths of the $\chi_{b}^{\prime}(J=2$ and $J=0)$ states relative to the $J=1$ state, the first observation of this decay might be expected in the transition between the $J=1$ states. $(d)$ Transitions at higher order in the multipole model, e.g. $Y(3 S) \rightarrow \omega \eta_{\mathrm{b}}(\mathrm{E} 1 \times \mathrm{E} 1 \times \mathrm{M} 1)$. $(e)$ Transitions to the $\eta_{b}$, which is accessible through two routes, each of which involves a radiative and an hadronic transition: either $Y(3 S) \rightarrow \pi \pi h_{b}\left(1^{1} P_{1}\right)$; $\mathrm{h}_{\mathrm{b}}\left(1^{1} \mathrm{P}_{1}\right) \rightarrow \gamma \eta_{\mathrm{b}}$, or $Y(3 \mathrm{~S}) \rightarrow \gamma \chi_{\mathrm{b}}^{\prime} ; \chi_{\mathrm{b}}^{\prime} \rightarrow \pi \pi \eta_{\mathrm{b}}$.

\section{DIRECT DECAYS OF BOTTOMONIA INTO HADRONS, QUARK VS GLUON FRAGMENTATION}

The comparison of total widths of bottomonia with predictions was covered admirably in a previous issue of this journal (116); here we touch briefly on some of the experimental advances since that time.

${ }^{13}$ For this $\mathbf{S} \rightarrow \mathbf{P}$ transition, Kuang \& Yan predict a dipion mass distribution that peaks at low values of invariant mass. This is understood by the following argument: such a transition $1^{-} \rightarrow 0^{+} 1^{+}$can only proceed in $P$ wave, which suppresses the high mass region. 
The $\chi_{\mathbf{b}}^{\prime} \mathrm{P}$ states provide us with a source of gluon jets, affording a direct comparison with $q \bar{q}$ jets at the same energy. Differences between ggg and q $\bar{q}$ events in the $10-\mathrm{GeV}$ center-of-mass energy range are well documented (122). The baryon rate per event from ggg decays of the $Y(1 S)$ is enhanced by a typical factor of two relative to the continuum, for example. On the other hand, no signal for open charm from the $r(1 S)$ has yet been observed (119). The three-gluon decays of the $Y$ 's are found to be more spherical than the more jet-like $q \bar{q}$ events, as expected by both the higher parton multiplicity as well as the selfcoupling property of the gluons. The event multiplicity, however, is only slightly larger for ggg decays than $q \bar{q}$ continuum events at $10 \mathrm{GeV}$. This is cortrary to expectations based on color considerations alone (117), which argue that the multiplicity for jets from gluons relative to quarks should be larger by a factor of $9 / 4$ at very high energies $\left(E_{C M}\right.$ $\rightarrow M_{\mathrm{Z}}$ ), retlecting the ratio of the color charge of the gluon to that of the quark. Higher order calculations reduce this value of $9 / 4$ by $\sim 15 \%$ at $W=10 \mathrm{GeV}(118)$.

Direct comparison of the two-parton fragmentation processes: $\mathrm{gg} \rightarrow$ hadrons, compared with $\mathrm{q} \overline{\mathrm{q}} \rightarrow$ hadrons, was performed recently by the CLEO collaboration (122). CLEO measures both the charged multiplicity and R2 distributions ${ }^{14}(121)$ for the three $J$ states by determining the number of photon tags corresponding to $Y(3 S) \rightarrow \gamma \chi_{b}^{\prime}(J=2,1$, $0)$. The multiplicity or R2 of each of the $\chi_{b}^{\prime}$ states is thereby mapped out. Data taken at the $Y(1 S)$ and the continuum allow the desired comparison of $\mathrm{gg}$, ggg, $q \overline{\mathrm{q}}$, and $\mathrm{q} \overline{\mathrm{q}} \mathrm{g}$ topologies, as shown in Figure 10. The values of mean observed charged multiplicity of the $J=(2,1,0)$ states are $(9.339 \pm 0.090 \pm 0.045,8.679 \pm 0.090 \pm 0.048,9.217 \pm 0.160 \pm$ 0.072).

These results are at variance with the naive expectation that twoparton gg jets will look very similar to $\mathrm{q} \overline{\mathrm{q}}$ jets at the same center-ofmass energy. The CLEO results indicate that the shape distributions given by the $J=0$ and $J=2$ decays are more consistent with $Y(1 S)$ decays than $q \bar{q}$ decays in their event characteristics, and that the net $\chi_{\mathrm{b}}^{\prime}$ shape is not far from the mean of the observed q $\bar{q}$ and $Y(1 S) \rightarrow$ ggg shape distributions. Also observed are similar multiplicity distributions for the $J=0$ and 2 states as compared with the $J=1$ state, at variance with the expectation based on color charge considerations that the multiplicity of gluon jets should exceed that of quark jets by a factor of $\sim 2$.

${ }^{14} \mathrm{R} 2$ correlates with thrust- $\mathrm{R} 2=1$ corresponds to a perfect two-jet event, $\mathrm{R} 2=0$ corresponds to a perfectly spherical event. 


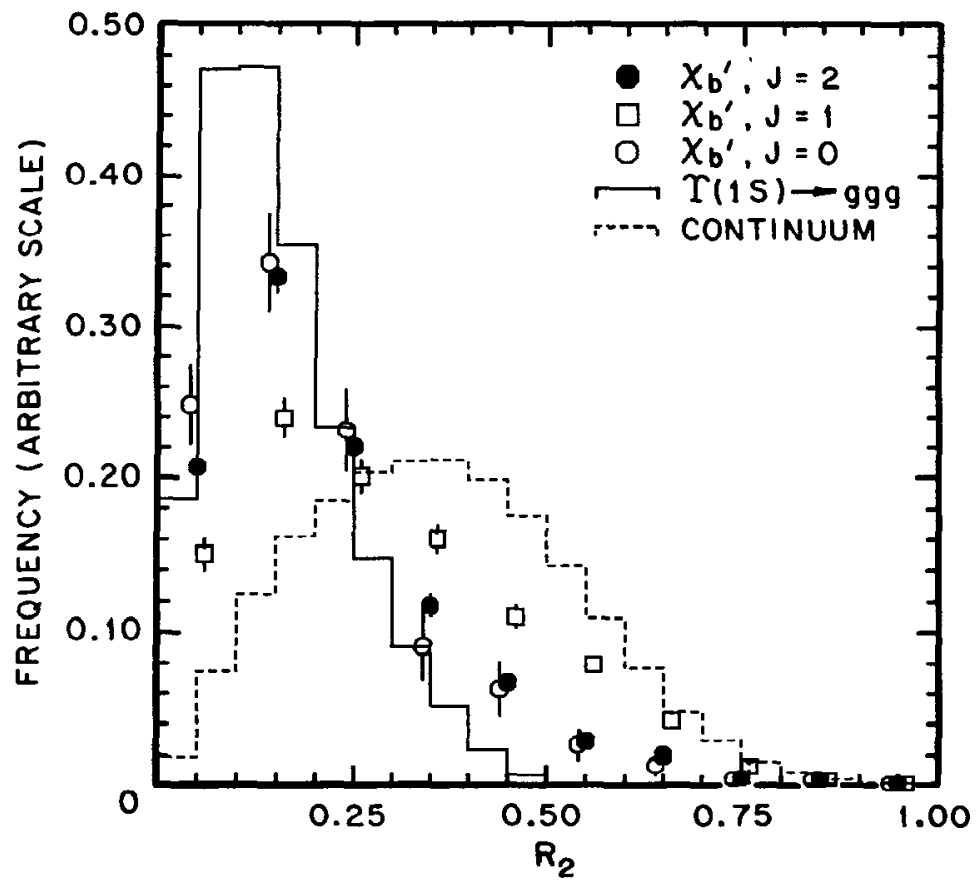

Figure $10 \mathrm{R} 2$ distributions for $\chi_{b}^{\prime}$ states, $\mathrm{q} \overline{\mathrm{q}}$, and (1S) events (122).

\section{RADIATIVE $Y(1 S)$ DECAYS}

\subsection{Measurement of $\alpha_{\mathrm{s}}$ from $Y(I S) \rightarrow g g \gamma$}

Measurement of the branching ratio $Y(1 S) \rightarrow \gamma g g$ relative to $Y(1 S) \rightarrow$ ggg is, as one might expect, somewhat insensitive to higher order $\alpha_{\mathrm{s}}$ corrections owing to the "cancellation" of two strong vertices (128). The rate can be written as

$$
B_{\mathrm{gg} \gamma}=\frac{\Gamma(Y \rightarrow \gamma \mathrm{gg})}{\Gamma(Y \rightarrow \mathrm{ggg})}=\frac{36}{5} e_{\mathrm{b}}^{2} \frac{\alpha}{\alpha_{\mathrm{s}}}\left[1+(2.2 \pm 0.6) \frac{\alpha_{\mathrm{s}}}{\pi}\right] .
$$

From $\alpha_{\mathrm{s}}$, one can infer the QCD scale parameter $\Lambda \overline{\mathrm{MS}}$ from the usual expressions, provided one has a prescription for the proper value of energy scale at which to evaluate the process (128).

Since there are typically $4-5 \pi^{0}$ 's produced in each $Y(1 S)$ decay, and since the $\operatorname{gg} \gamma$ branching ratio is typically $2 \%$, the momentum range measured for the direct photon spectrum is limited by the ability to separate the background photons from $\pi^{0} \rightarrow \gamma \gamma$ (and $\eta \rightarrow \gamma \gamma$ ) from the 
signal photons of interest. Discrimination against these backgrounds becomes difficult for scaled photon momenta less than $25 \%$ of the available center-of-mass energy [ $\sim 2.4 \mathrm{GeV}$ for $Y(1 \mathrm{~S})$ decays].

Combining the experimental measures (123), we find $B_{\mathrm{gg} \gamma}=2.77 \pm$ $0.15 \%$, corresponding to a value of $\alpha_{\mathrm{s}}\left(Q^{2}=2.2 \mathrm{GeV}^{2}\right) \cong 0.25$. Excepting the earliest measurement (124), it is by now well established that the photon spectrum from $Y(1 S) \rightarrow$ ygg is not that predicted by lowest order QCD (123). The agreement with the phenomenological model of Field (125), which takes into account the effects on the photon spectrum due to hadronization in the recoil gluon system, is actually quite good. What remains to be done experimentally is to verify the predictions for the photon angular spectrum as a function of photon momenturn, and to survey the gg system recoiling against the photon, with the intent of comparing inclusive particle yields, event shapes, etc with ggg events of a comparable topology.

\subsection{Exclusive Radiative Decays}

The radiative decays of the $Y(1 S)$ can provide a door to a host of exotica, including higgs bosons, cosmions ${ }^{15}$ (129) and other noninteracting particles, glueballs, and axions. For the "standard" axion, the axion-heavy quark couplings are prescribed by a single parameter $x$, the ratio of the vacuum expectation values of the Higgs fields. For $q \bar{q}$ decaying radiatively into $\gamma+$ axion, the dependence of branching ratio on $x$ is proportional to $x^{2}\left(x^{-2}\right)$ for charge $2 / 3(-1 / 3)$ quarks. Hence, the product branching ratio $B(\psi \rightarrow \gamma+a) \times B(Y \rightarrow \gamma+a)$ is independent of $x$.

The observation of resonances in the mass spectrum opposite photons is also one of the ways of establishing or refuting possible glueball candidates. Studies by the MARK III and DM2 collaborations provide a detailed list of two-body radiative $\psi$ decays. Although, in many cases, the particles recoiling against the photon are "ordinary" ( $\eta$ 's, for example) in some cases, the particles are "exotic" [ $\eta(1440)$, for example] or familiar particles $\left[f_{2}(1270)\right.$, for example] with masses close to those expected from lattice calculations for glueballs with similar quantum numbers. In particular, the nonobservation of the $\eta(1440)$ in the reaction $\gamma \gamma \rightarrow \eta(1440)$ strengthens the notion that this object is a glueball-if it were predominantly quark matter, there should be an observable two-photon coupling to the charges. Combining this with

\footnotetext{
15 "Cosmion" is a generic term referring to a particle residing in the solar interior that can act as a heat sink, and thus lower the temperature of the solar interior sufficiently so as to decrease significantly the expected rate of ${ }^{8} \mathrm{~B}$ neutrinos.
} 
information from decays where an $\omega$ or a $\phi$ replaces the photon, one is able to determine the quark composition of a particular object, and, by inference, its glue fraction as well.

Exclusive multibody radiative $Y$ decays have been seen (127), and the sensitivities in $Y$ decay are now of the order of 100 times greater than the level at which these same decays were seen from the $\psi$. Nevertheless, no convincing signals have yet been observed for two-body radiative $Y(1 S)$ decays, and the efforts to observe the glueball candidates seen in radiative $\psi$ decay have so far been unsuccessful. There have been many searches for other exotica, with many null results. The standard axion is ruled out by the combined results of radiative $\psi$ and radiative $Y$ decay, as is a neutral higgs particle with $m_{\mathrm{h}}<5 \mathrm{GeV}$ (much more stringent limits have been set since by LEP). For a noninteracting particle X (cosmion, SUSY particle, etc) coupling through an axial vector-axial vector current to bottomonia in the reaction $Y(1 S)$ $\rightarrow \gamma \overline{\mathrm{X} X}$, limits of the order $4 \times 10^{-5}$ have been established (126). In conjunction with direct WIMP searches, this requires $m_{\mathrm{X}}>2 \mathrm{GeV}$ to help explain the observed ${ }^{8} \mathrm{~B}$ solar neutrino deficit in the chlorine experiments. For $X$ with vector-vector couplings produced via $Y(1 S) \rightarrow$ $\overline{X X}$, the present limit of 0.05 , determined by the Particle Data Group (89), requires a factor of 50 more sensitivity to rule out the cosmion explanation of the solar neutrino deficit.

\section{SUMMARY}

Even though we have already learned quite a bit from the study of upsilon spectroscopy, and experimental progress has been significant in the last decade, the portrait of upsilon spectroscopy is still somewhat impressionistic. Aside from the narrow $Y$ resonances and the two $\chi_{\mathrm{b}}$ triplets below threshold, no other bottomonia states have been observed. This is in contrast to the $\psi$ system, where the $\eta_{c}$ is well established and the singlet $h_{c}$ is an increasingly well-studied object. Part of this discrepancy is a consequence of the generally smaller production rates and higher combinatoric backgrounds one must contend with in bottomonia. The $p \bar{p}$ gas jet annihilation experiment E760, for instance, which has recently provided essential information on charmonia, will not have the same success for $b \bar{b}$ because the $b \bar{b} \rightarrow \bar{p} p$ cross section is so small. The $b \bar{b} \mathrm{D}$ states, the dipion transition $Y(3 S) \rightarrow \chi_{\mathrm{b}}^{\prime} \gamma, \chi_{\mathrm{b}}^{\prime} \rightarrow$ $\chi_{\mathrm{b}} \pi \pi$, and radiative decays of the $\chi_{\mathrm{b}}(J=0)$ states can probably be observed with another factor of 5 increase over the world's present sample of $\mathrm{e}^{+} \mathrm{e}^{-} \rightarrow Y(3 \mathrm{~S})$ events. However, the task of unambiguous determination of all of the above, as well as the observation of vibra- 
tional states in bottomonium and of hadronic and radiative transitions that are higher order than electric dipole, will almost certainly have to wait for the B-factory era.

\section{ACKNOWLEDGMENTS}

We wish to thank our colleagues on the CLEO, CUSB, ARGUS, and Crystal Ball experiments for providing us with their time, help, and direct input to the text. In particular, we wish to thank Jay Dominick, Jim Fast, Manoj Thulasidas, Giancarlo Moneti, and Qiuwen Wu.

\section{Literature Cited}

1. Aubert JJ, et al:!Phys. Rev. Lett. 33: 1404 (1974); Augustì JE, et al. Phys. Rev. Lett. 33:1406:(1974)

2. Herb SW, et al. Phys. Rev. Lett. 39: 252 (1977); Innes WR; et al. Phys. Rev. Lett. 39:1240, 1640 (1977)

3. Abe F, et al. (CDF).Phys. Rev. Lett. 68:447 (1992)

4. Kühn JHE, Ono S. Z. Phys. C21:395 (1984); C24:104 (1984); Kühn JH Acta Phys. Polon. B16:969 (1985); Kwong W. Phys, Rev. D43:1488 (1991)

5. Berger Ch, et al. Phys. Lett. B76:243 (1978); Dardeen CW, et al. Phys. Lett. B76:246 (1978); Bienlein JK, et al. Phys. Lett. B76:360 (1978)

6. Andrews D, et al. Phys. Rev. Lett. 44: 1108 (1980); Böhringer T, et al. Phys. Rev. Lett. 44:1111 (1980); Andrews D, et al. Phys. Rev. Lett. 45:219 (1980); Finocchiaro G, et al. Phys. Rev. Lett. 45:222 (1980); and Ref. 16

7. Franzini P, Lee-Franzini J. Annu. Rev. Nucl. Part. Sci. 33:1 (1983)

8. Lee-Franzini, J. Nucl. Instrum. Methods A263:35 (1988); Tuts PM. Nucl. Instrum. Methods A265:243 (1988)

9. Bloom E, Peck C. Annu. Rev. Nucl. Part. Sci. 33:143 (1983); Oreglia M, et al. (Crystal Ball). Phys. Rev. D25: 2259 (1982)

10. Schamberger RD, et al (CUSB-II). Nucl. Instrum. Methods A309:450 (1991)

11. Albrecht $\mathrm{H}$, et al (ARGUS). Phys. Lett. B134:137 (1984)

12. Andrews D, et al (CLEO). Nucl. Instrum. Methods A211:47 (1983)

13. Kubota Y, et al (CLEO-II). Nucl. Instrum. Methods A320:66 (1992)

14. Alexander J, et al. Phys. Rev. Lett. B64:2226 (1990)

15. Danilov M. In Proc. Int. Lepton. Photon Symp. and Europhysics Conf. on High Energy Physics, ed. S
Hegarty, K Potter, E Quercigh, p. 331. World Sci. Publ. (1992)

16. Lovelock DMJ, et al (CUSB). Phys. Rev. Lett. 54:377 (1985); Besson D, et al (CLEO). Phys. Rev. Lett. 54:381 (1985); Akerib DS, et al (CLEO-II) Phys. Rev. Lett. 67:1692 (1991)

17. Barbieri R, et al. Phys. Lett. B57:455 (1975); Barbieri R, Gatto R, Remiddi E. Phys. Lett. B61:465 (1976); Barbieri R, Caffo M, Remiddi E. Nucl. Phys. B162:220 (1980)

18. Jakubowski Z, et al (Crystal Ball). $Z$. Phys. C40:49 (1988)

19. Niczyporuk B, et al (LENA). Phys. Lett. B99:169 (1981)

20. Albrecht $\mathrm{H}$, et al (DASP). Phys. Lett. 116:B83 (1982); Niczyporuk B, et al (LENA). Z. Phys. C15:299 (1982); Bock P, et al (DESY-HD). Z. Phys. C6:125 (1980); Berger $\mathrm{Ch}$, et al (PLUTO). Z. Phys. C1:343 (1979)

21. Giles R, et al (CLEO). Phys. Rev. D29:1285 (1984)

22. Serednyakov SI, et al. Zh. Eksp. Teor. Fiz. 71:2025 (1976) [Sov. Phys. JETP 44:1063 (1976)]; Derbenev YaS, et al. Part. Accel. 10:177 (1980)

23. Baru SE, et al (VEPP-4). Z. Phys. C30:551 (1986); MacKay WW, et al (CESR, CUSB). Phys. Rev. D29:2483 (1984); Artamonov AS, et al (VEPP4). Phys. Lett. B137:272 (1984); Baru SE, et al (VEPP-4). Z. Phys. C32:662 (1986); Barber DP, et al (DORIS, ARGUS, Crystal Ball). Phys. Lett. B 135:498 (1984)

24. Kobel M, et al (Crystal Ball). Z. Phys. C53:193 (1992)

25. Chen W-Y, et al (CLEO). Phys. Rev. D39:3528 (1989); Kaarsberg T, et al (CUSB-II). Phys. Rev. Lett. 62:2077 (1989); Lee-Franzini J (CUSB). Nucl. Phys. (Proc. Suppl.) 3:139 (1988); Andrews D, et al (CLEO). Phys. Rev. Lett. 50:807 (1983); Albrecht H, et al (DASP). Phys. Lett. 116:B83 (1982); 
Niczyporuk B, et al (LENA). $Z$. Phys. C15:299 (1982); Bock P, et al (DESY-HD). Z. Phys. C6:125 (1980); Albrecht $\mathrm{H}$, et al (ARGUS). Z. Phys. C28:45 (1985)

26. Giles R, et al (CLEO). Phys. Rev. Lett. 50:877 (1983); Albrecht $\mathrm{H}$, et al (ARGUS). Phys. Lett. B154:452 (1985)

27. Albrecht $\mathrm{H}$, et al (ARGUS). Z. Phys. C35:283 (1987); and Ref. 102

28. Tsai YS. SLAC preprint SLAC-PUB3129 (1983); Cooper S. In Proc. 23rd Int. Conf. of High Energy Phys., Berkeley, July 1986, ed. SC Loken, p. 92. World Sci. Publ. (1987); Koenigsmann K. DESY preprint, DESY 86-136 (1986); Baru SE, et al. Z. Phys. C30:551 (1986); Alexander $\mathbf{J}$, et al. Nucl. Phys. B320:45 (1989)

29. Van Royen R, Weisskopf V. Nuovo Cimento A50:617 (1967)

30. Barbieri R, et al. Phys. Lett. B57:455 (1975); Celmaster W. Phys. Rev. D19:1517 (1979)

31. Mackenzie PB, Lepage GP. Phys. Rev. Lett. 47:1244 (1981); Kwong W, et al. Phys. Rev. D28:228 (1983)

32. Duke DW, Roberts RG. Phys. Rep. 120:275 (1985); and Ref. 128

33. Hikasa K, et al (Particle Data Group). Phys. Rev. D45:1 (1992)

34. Klopfenstein C, et al (CUSB). Phys. Rev. Lett. 51:160 (1983)

35. Haas P, et al (CLEO). Phys. Rev. Lett. 52:799 (1984)

36. Nernst R, et al (Crystal Ball). Phys. Rev. Lett. 54:2195 (1985)

37. Albrecht H, et al (ARGUS). Phys. Lett. B160:331 (1985)

38. Pauss F, et al (CUSB). Phys. Lett. B130:439 (1983)

39. Walk W, et al (Crystal Ball). Phys. Rev. D34:2611 (1986)

40. Skwarnicki T, et al (Crystal Ball). Phys. Rev. Lett. 58:972 (1987); and Ref. 41

41. Skwarnicki $\mathrm{T}$. PhD thesis, Inst. for Nucl. Phys., Krakow (1986); DESY Internal Rep. DESY F31-86-02 (1986)

42. Morrison R, et al (CLEO-II). Phys. Rev. Lett. 67:1696 (1991)

43. Han K, et al (CUSB). Phys. Rev. Lett. 49:1612 (1982)

44. Narin M, et al (CUSB-II). Phys. Rev. Lett. 66:3113 (1991)

45. Crawford G, et al (CLEO-II). Phys. Lett. B294:139 (1992)

46. Heintz U, et al (CUSB-II). Phys. Rev. Lett. 66:1563 (1991)

47. Gaiser J, et al (Crystal Ball). Phys. Rev. D34:711 (1986); Edwards C, et al (Crystal Ball). Phys. Rev. Lett. 48: 70 (1982)

48. Fulcher LP. Phys. Rev. D42:2337 (1990)

49. Lichtenberg DB, et al. Z. Phys. C41: 615 (1989)

50. Fulcher LP. Phys. Rev. D37:1259 (1988)

51. Fulcher LP. Phys. Rev. D44:2079 (1991)

52. Gupta SN, Repko WW, Suchyta CJ III. Phys. Rev. D39:974 (1989)

53. Büchmuller W, Grunberg G, Tye SH. Phys. Rev. Lett. 45:103 (1980). Phys. Rev. D24:132 (1981)

54. Moxhay P, Rosner JL. Phys. Rev. D28:1132 (1983)

55. Richardson JL. Phys. Lett. 82:B72 (1979)

56. Gupta SN, Radford SF, Repko WW. Phys. Rev. D26:3305 (1982); D30: 2424 (1984); see also Ref. 57

57. Gupta SN, Radford, SF, Repko, WW. Phys. Rev. D34:201 (1986)

58. Bhanot G, Rudaz S. Phys. Lett. 78: B19 (1978)

59. Ito H. Prog. Theor. Phys. 84:94 (1990)

60. Bander M, et al. Phys. Lett. 134:B58 (1984); Phys. Rev. D29:2038 (1984); and Ref. 61

61. Daghighian F, Silverman D. Phys. Rev. D36:3401 (1987)

62. Quigg C, Rosner JL. Phys. Lett. 71: B53 (1977); Phys. Rep. 56:167 (1979)

63. Eichten E, Feinberg F. Phys. Rev. D23:2724 (1981)

64. Krasemann H, Ono S. Nucl. Phys. 154:B83 (1979)

65. McClary R, Byers N. Phys. Rev. D28:1692 (1983)

66. Eichten E, et al. Phys. Rev. D21:203 (1980)

67. Quigg C, Rosner JL. Phys. Rev. D23: 2625 (1981)

68. Crater H, Van Alstine P. Phys. Rev. Lett. 53:1527 (1984)

69. Grotch H, Owen DA, Sebastian KJ. Phys. Rev. D30:1924 (1984)

70. Martin M. Phys. Lett. 100:B11 (1981)

71. Grant AK, Rosner JL, Rynes E. Enrico Fermi Inst, preprint EFI-92-42 (1992)

72. Heikkilä $\mathrm{K}$, Tórnqvist NA, Ono S. Phys. Rev. D29:110 (1984); Z. Phys. C23:59 (1984)

73. Mackenzie PB. Presented at Lattice 92, Amsterdam, Sept. 15-19, 1992, Fermilab preprint FERMILABCONF-92/373-T (1992); Lepage GP. In Lattice 91, Nucl. Phys. B26:45 (1992) 
74. Ito H. Prog. Theor. Phys. 74:1092 (1985)

75. Kwong W, Rosner JL. Phys. Rev. D38:279 (1988)

76. Fulcher LP. Phys. Rev. D39:295 (1988)

77. Kwong W, et al. Phys. Rev. D37:3210 (1988)

78. Bodwin CiT, Braaten E, Lepage GP. Phys. Rev. Rapid Commun. D46: R1914 (1992)

79. de Forcrand P, Stack JD. Phys. Rev. Lett. 55:1254 (1985); Huntley A, Michael C. Nucl. Phys. B286:211 (1987); Campostrini M, Moriarty K, Rebbi C. Phys. Rev. Lett. 57:44 (1986); Phys. Rev. D36:3450 (1987); Koike Y. Phys. Rev. Lett. 59:962 (1987); Phys. Lett. B216:184 (1989)

80. Armstrong TA, et al (E760), Fermilab preprint FERMILAB-PUB-92-186-E (1992)

81. Gromes D. Z. Phys. C26:401 (1984)

82. Hirano M. Prog. Theor. Phys. 83:575 (1990)

83. Carlson J, Kogut JB, Pandharipande VR. Phys. Rev. D28:2807 (1983)

84. Büchmuller W. Phys. Lett. 112:B79 (1982)

85. Dib CO, Gilman JG, Franzini PJ. Phys. Rev. D37:735 (1988)

86. Franzini P. Phys. Lett. B296:195 (1992); model E Phys. Lett. B296:199 (1992)

87. Hiller JR. Phys. Rev. D30:1520 (1984)

88. Pantaleone J, Tye S-H, Ng YJ. Phys. Rev. D33:777 (1986)

89. The Particle Data Group, Phys. Rev. D45:I.1 (1992)

90. Abrams G, et al. Phys. Rev. Lett. B347:118! (1975)

91. Gottfried K, in Proc. Int. Symp. on Lepton and Photon Interactions at High Energies, Hamburg, ed. F Gutbrod. Hamburg: DESY (1977), p. 667; Phys. Rev. Lett. B40:598 (1978)

92. Yan TM. Phys. Rev. D22:1652 (1980)

93. Voloshin MB. Nucl. Phys. B154:365 (1979); Voloshin MB, Zakharov V. Phys. Rev. Lett. B45:688 (1980)

94. Novikov VA, Shifman MA. Z. Phys. 8:43 (1981)

95. Voloshin MB. Pis'ma Zh. Eksp. Teor. Fiz. 37:58 (1983) [JETP Lett. 37:69 (1983)]

96. Kuang YP, Yan TM. Phys. Rev. D24: 2874 (1981)

97. Zhou HY, Kuang YP. Phys. Rev. D44:756 (1991)

98. Dominick JM. Hadronic $\pi^{+} \pi^{-}$Transitions in $Y(3 S)$ Decays, $\mathrm{PhD}$ thesis Purdue Univ. (1993) (unpublished)
99. Thulasidas M. Hadronic $\pi^{0} \pi^{0}$ Transitions in $\mathrm{Y}(3 S)$ Decays. $\mathrm{PhD}$ thesis, Syracuse Univ. (1993) (unpublished)

100. Wu OW, et al. Phys. Lett. B301:307 (1993)

101. Albrecht H, et al (ARGUS). Z. Phys. 35:283 (1987)

102. Besson D, et al (CLEO). Phys. Rev. D30:1433 (1984)

103. Fonseca V, et al (CUSB). Nucl. Phys. B242:31 (1984)

104. Gelphman G, et al (Crystal Ball). Phys. Rev. D11:2893 (1985)

105. Brock IC, et al (CLEO). Phys. Rev. D43:1448 (1991)

106. Brown LS, Cahn RN. Phys. Rev. Lett. B35:1 (1975)

107. Cahn RN. Phys. Rev. D12:3559 (1975)

108. Chakravarty S, Ko P, Univ. Minn. preprint UMN-TH-1115 (1992)

109. Bélanger $G$, DeGrand T, Moxhay $P$. Phys. Rev. D39:257 (1989)

110. Morgan D, Pennington MR. Phys. Rev. D12:1283 (1975)

111. Coffou E, Martinis M, Mikuta-Martinis V. Phys, Rev. D33:136 (1986)

112. Lipkin HJ, Tuan SF. Phys. Lett. B206:349 (1988)

113. Moxhay P. Phys. Rev. D39:3497 (1989)

114. Deleted in proof

115. Armstrong TA, et al (the E760 collaboration). Phys. Rev. Lett. B69: 2337 (1992)

116. Kwong W, Rosner JL, Quigg C. Annu. Rev. Nucl. Part. Sci. 37:32582 (1987)

117. Einhorn MB, Weeks BG. Nucl. Phys. B146:445 (1978); Shizuya K, Tye SH. Phys. Rev. Lett. B41:787 (1978)

118. Gaffney JB, Mueller AH. Nucl. Phys. B250:109 (1985)

119. Albrecht H, et al. Search for Charm Production in Direct Decays of the $Y(I S)$ Resonance, DESY 92-008 (1992). Z. Phys. C55, p. 25. (1992)

120. Deleted in proof

121. Fox GC, Wolfram S. Phys. Rev. Lett. B41:1581 (1978); Phys. Lett. 82B:134 (1979)

122. Butler F, et al (CLEO). Phys. Rev. D46:4822 (1992)

123. Csorna $S$, et al (CLEO). Phys. Rev. Lett. B56:1222 (1986), Albrecht H, et al (ARGUS). Phys. Lett. B199:291 (1987); Bizzeti A, et al (Crystal Ball). Phys. Lett. B267:286 (1991)

124. Schamberger RD, et al. Phys. Lett. B138:225 (1984)

125. Field RD. Phys. Lett. B133:248 (1983) 
378 BESSON \& SKWARNICKI

126. Antreasyan D, et al. Phys. Lett. B251:204 (1990)

127. Fulton R, et al. Phys. Rev. D41:1401 (1990)
128. Brodsky S, Lepage GP, Mackenzie P. Phys. Rev. D28:I.228 (1983.)

129. Fayet P, Kaplan J. Phys. Lett. B269: 213 (1991) 